\title{
Anti-Inflammatory Effects of Heritiera littoralis Fruits on Dextran Sulfate Sodium- (DSS-) Induced Ulcerative Colitis in Mice by Regulating Gut Microbiota and Suppressing NF- $\kappa$ B Pathway
}

\author{
Guosheng Lin $\mathbb{D}^{1},{ }^{1}$ Minyao $\mathrm{Li}^{2}{ }^{2}$ Nan Xu, ${ }^{2}$ Xiaoli Wu $\mathbb{D}^{3},{ }^{3}$ Jingjing Liu, ${ }^{2}$ Yulin $\mathrm{Wu},{ }^{2}$ \\ Qian Zhang, ${ }^{4,5}$ Jian Cai, ${ }^{4,5}$ Changjun Gao $\mathbb{C}^{4,5}$ and Ziren Su $\mathbb{1}^{2}$ \\ ${ }^{1}$ Research Center of Chinese Herbal Resource Science and Engineering, Key Laboratory of Chinese Medicinal Resource from Lingnan, \\ Ministry of Education, Guangzhou University of Chinese Medicine, Guangzhou 510006, China \\ ${ }^{2}$ School of Pharmaceutical Science (Mathematical Engineering Academy of Chinese Medicine), Guangzhou University of \\ Chinese Medicine, Guangzhou 510006, China \\ ${ }^{3}$ School of Biomedical and Pharmaceutical Sciences, Guangdong University of Technology, Guangzhou 510006, China \\ ${ }^{4}$ Guangdong Academy of Forestry, Guangzhou 510520, China \\ ${ }^{5}$ Guangdong Provincial Key Laboratory of Silviculture, Protection and Utilization, Guangzhou 510520, China
}

Correspondence should be addressed to Changjun Gao; gaochangjun015@163.com and Ziren Su; suziren@gzucm.edu.cn

Received 3 September 2020; Revised 13 November 2020; Accepted 27 November 2020; Published 7 December 2020

Academic Editor: Mohammad Hassan Baig

Copyright $\odot 2020$ Guosheng Lin et al. This is an open access article distributed under the Creative Commons Attribution License, which permits unrestricted use, distribution, and reproduction in any medium, provided the original work is properly cited.

\begin{abstract}
Aim of the Study. This study is aimed at exploring the effects and pharmacological mechanisms of the extracts from the Heritiera littoralis fruit (EFH) on dextran sulfate sodium- (DSS-) induced ulcerative colitis (UC) in mice. Materials and Methods. The chemical compositions of EFH were identified using LC-ESI-MS. The mice with 3\% DSS-induced UC were administered EFH $(200,400$, and $800 \mathrm{mg} / \mathrm{kg}$ ), sulfasalazine (SASP, $200 \mathrm{mg} / \mathrm{kg}$ ), and azathioprine (AZA, $13 \mathrm{mg} / \mathrm{kg}$ ) for 10 days via daily gavage. The colonic inflammation was evaluated by the disease activity index (DAI), colonic length, histological scores, and levels of inflammatory mediators. The gut microbiota was characterized by $16 \mathrm{~S}$ rRNA gene sequencing and analysis. Results. LC-ESI-MS analysis showed that EFH was rich in alkaloids and flavones. The results indicated that EFH significantly improved the DAI score, relieved colon shortening, and repaired pathological colonic variations in colitis. In addition, proteins in the NF- $\kappa \mathrm{B}$ pathway were significantly inhibited by EFH. Furthermore, EFH recovered the diversity and balance of the gut microbiota. Conclusions. EFH has protective effects against DSS-induced colitis by keeping the balance of the gut microbiota and suppressing the NF- $\kappa$ B pathway.
\end{abstract}

\section{Introduction}

Ulcerative colitis (UC) belongs to the inflammatory bowel disease (IBD) family and is a nonspecific chronic inflammatory disease (Do et al., 2020). UC clinically appears as chronic diarrhea, mucous-filled and bloody purulent stools, abdominal pain, weight loss, and fatigue [1], which all seriously affect people's quality of life. The current treatments for UC are aminosalicylic drugs, glucocorticoid drugs, immunosuppressants, and monoclonal antibodies, but these drugs cannot achieve ideal results because of the unclear etiology of UC [2]. As the morbidity of UC and the incidence of disease flares worsen, people increasingly turn to alternative medicine approaches [3], so there is a growing need to develop novel, efficient, and safe candidates for UC treatment.

Heritiera littoralis Dryand. (Sterculiaceae), a semimangrove plant, is typically found in mangrove zones, which are mostly tropical and subtropical areas [4]. Mangroves not only protect the environment but also have medicinal value. In terms of medicinal uses, Heritiera littoralis Dryand. 
TABLE 1: Sequences of primers used for QRT-PCR.

\begin{tabular}{lccc}
\hline Gene & & Primer sequences $\left(5^{\prime}-3^{\prime}\right)$ & Product size (bp) \\
\hline \multirow{2}{*}{ iNOS } & Reverse & CAGCCACATTGATCTCCGTGACAG & 358 \\
& Forward & GATGTGCTGCCTCTGGTCTTGC & 253 \\
COX-2 & Reverse & GCGGTTCTGATACTGGAACTGCTG & 22 \\
& Forward & TGGTCTGGTGCCTGGTCTGATG & 22 \\
IL-17 & Reverse & GGTCTTCATTGCGGTGGAGAGTC & 191 \\
& Forward & TGATGCTGTTGCTGCTGCTGAG & 378 \\
IL-4 & Reverse & CGAAAGAGTCTCTGCAGCTCCA & 378 \\
& Forward & GTCACAGGAGAAGGGACGCC & 235 \\
IL-12 & Reverse & GCAGACAGAGACGCCATTCCAC & \\
& Forward & CACCTGTGACACGCCTGAAGAAG & \\
\end{tabular}

has been used to treat indigestion, diarrhea, and dysentery; the medicinal parts are mainly in the seeds and are obtained by decoction [5]. Existing literature had reported that the Heritiera littoralis bark exerted its anti-inflammatory effects by reducing the release of NO, PGE2, and TNF- $\alpha$, as well as by downregulating iNOS and COX-2 [6]. In addition, Heritiera littoralis leaf extracts have exhibited obvious antibacterial and anticancer activities [7, 8]. The pharmacodynamics of the Heritiera littoralis fruit has not been clearly reported in publications, but the fruit does contain antiinflammatory chemicals, such as flavonoids and triterpenoids [9]. Therefore, we hypothesized that the extracts of the Heritiera littoralis fruit (EFH) may have antiinflammatory effects.

In this study, we proposed that UC is caused by the imbalance of intestinal microbiota, associated with intestinal inflammation responses [10]. NF- $\kappa \mathrm{B}$ contributes to the mechanism of the inflammatory process and controls the release of inflammatory cytokines involved in UC [11]. Therefore, we hypothesized that gut microbiota dysbiosis would improve after introduction of EFH to the intestinal microenvironment, as a result of changes to the release of inflammatory cytokines and inhibition of the NF- $\kappa \mathrm{B}$ pathway. In our research, we investigated the mechanism of action for EFH in dextran sulfate sodium- (DSS-) induced colitis in an experimental murine model.

\section{Materials and Methods}

2.1. Materials. The Heritiera littoralis fruits were provided by the Guangdong Academy of Forestry and identified by Professor Jian Cai of that academy. The voucher specimens (914556) were deposited for further reference in South China Botanical Garden (Guangzhou, China). Dextran sulfate sodium was bought from MP Biomedicals (Montreal, Canada). Sulfasalazine (SASP) was provided by Shanghai Xinyi Tianping Pharmaceutical Co., Ltd. (Shanghai, China) and azathioprine (AZA) by Aspen Pharmacare Australia Co., Ltd, (Australia). The ELISA kits for TNF- $\alpha$, IFN- $\gamma$, IL$1 \beta$, and IL-6 were bought from the Shanghai MLBIO Biotechnology Co., Ltd. (Shanghai, China). The kit for bio- chemical analysis of myeloperoxidase (MPO) was obtained from the Nanjing Jiancheng Bioengineering Institute (Nanjing, Jiangsu, China). The antibodies (NF- $\kappa \mathrm{B}$ p65, NF$\kappa \mathrm{B}$ p-p65, I $\kappa \mathrm{B} \alpha, \mathrm{p}-\mathrm{I} \kappa \mathrm{B} \alpha, \mathrm{IKK} \alpha, \mathrm{p}$-IKK $\alpha, \beta$-actin, and Histone H3) were purchased from Affinity Biosciences (Ohio, USA). All other reagents and chemicals were of analytical grade.

2.2. Preparation of Extracts. The Heritiera littoralis fruits were collected from the Nansha Wetland Park (Guangdong, Guangzhou, China). The Heritiera littoralis fruits were dried at $60^{\circ} \mathrm{C}$, pulverized and filtered through an 80-mesh sieve to obtain a dry product for use. The dried fruits $(100 \mathrm{~g})$ were extracted by 20 -fold distilled water for $2 \mathrm{~h}$. After filtration, the residue was reextracted by 20 -fold distilled water under the same condition. Thereafter, the two filtrates were combined in a container and then evaporated to $100 \mathrm{~mL}$. Finally, the fruit extracts were stored in a refrigerator at a concentration of $1 \mathrm{~g} / \mathrm{mL}$ for subsequent animal experiments $[12,13]$.

2.3. LC-ESI-MS Analysis. The extracts of EFH for chemical composition analysis were precipitated by $95 \%$ ethanol. The filtrate was concentrated to dryness in vacuum and dissolved in methanol. The EFH was analyzed by liquid chromatography-electrospray ionization mass spectrometry (LC-ESI-MS) with ESI-MS-positive and ESI-MS-negative ion acquisition modes. The sample $(5 \mu \mathrm{L})$ was injected into the UPLC apparatus equipped with a reverse phase C-18 column $(150 \times 2.1 \mathrm{~mm} 1.8 \mu \mathrm{m}$, Welch $)$. Mobile phase elution was performed with a flow rate of $0.3 \mathrm{~mL} / \mathrm{min}$ using water acidified with $0.1 \%$ formic acid (A) and acetonitrile acidified with $0.1 \%$ formic acid (B), as follows: $0-1 \mathrm{~min}, 98-98 \% \mathrm{~A}$ $(v / v), 2-2 \%$ B $(v / v) ; 1-5$ min, $98-80 \%$ A, 2-20\% B; 5-10 min, $80-50 \%$ A, $20-50 \%$ B; $10-15 \min , 50-20 \%$ A, $50-80 \%$ B; $15-$ $20 \mathrm{~min}, 20-5 \%$ A, $80-95 \%$ B; $20-25$ min, $5-5 \%$ A, $95-95 \%$ B; and $25-26 \mathrm{~min}, 5-98 \% \mathrm{~A}, 95-2 \% \mathrm{~B}$; and $26-30 \mathrm{~min}, 98-98 \%$ A, $2-2 \%$ B. The positive ion mode was adjusted to a $300^{\circ} \mathrm{C}$ capillary temperature and $3 \mathrm{kV}$ capillary voltage. All data collected were acquired and processed by the MassLynx 4.1 software. 


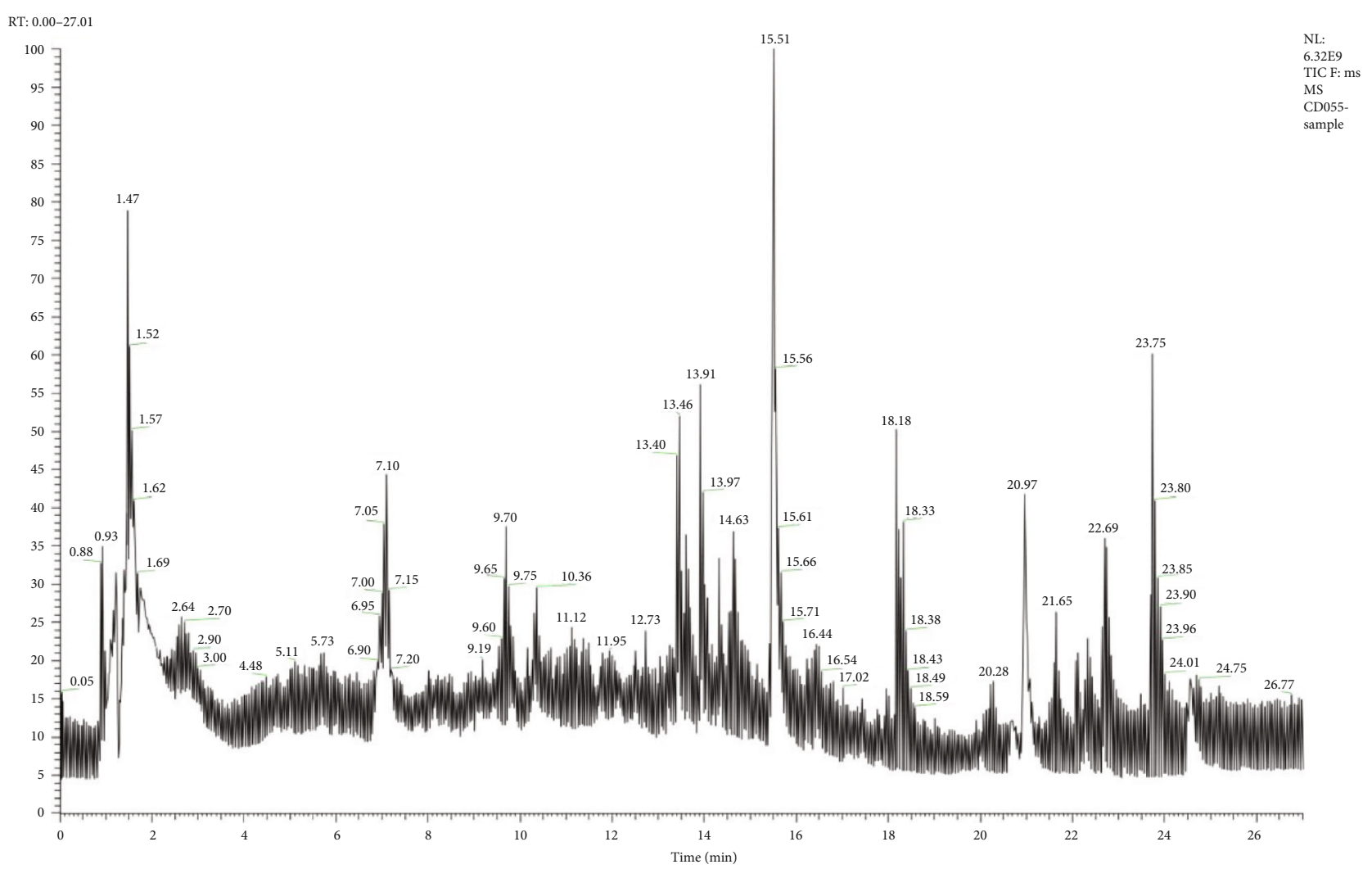

(a)

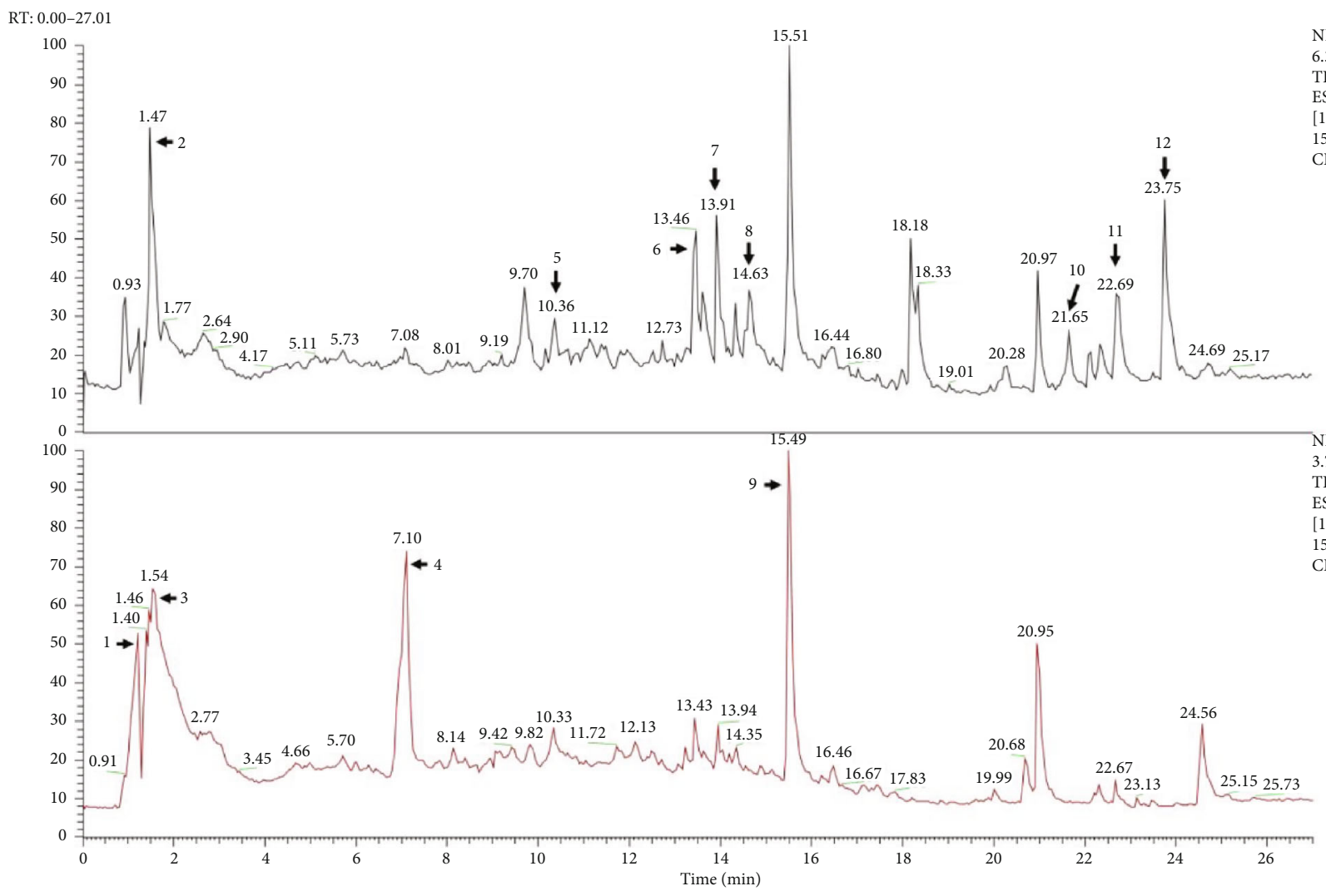

(b)

FIgure 1: The LC-ESI-MS chromatograms of EFH. (a) The total ion currents of EFH. (b) Positive mode in black and negative mode in red. Peak assignments are listed in Table 2. 
TABLE 2: The phytochemical constituents of EFH.

\begin{tabular}{|c|c|c|c|c|c|}
\hline Order & Retention time (R.t) & Components & Molecular weight & Area (\%) & Ref. \\
\hline 1 & 1.397 & L-glutamic acid & 147.05313 & $4.34 \%$ & {$[18]$} \\
\hline 2 & 1.471 & Triglycidyl glycerol & 277.15236 & $8.97 \%$ & {$[19]$} \\
\hline 3 & 1.539 & 2-Naphthalenesulfonic acid & 208.02116 & $10.82 \%$ & {$[20]$} \\
\hline 4 & 7.116 & Penicillic acid & 170.058 & $10.56 \%$ & {$[21]$} \\
\hline 5 & 10.361 & Syringic acid & 198.05271 & $0.92 \%$ & {$[22]$} \\
\hline 6 & 13.453 & Puerarin & 416.10993 & $1.99 \%$ & {$[23]$} \\
\hline 7 & 13.92 & Taxifolin & 304.05788 & $2.34 \%$ & {$[24]$} \\
\hline 8 & 14.621 & 4-Hydroxycoumarin & 162.03058 & $1.32 \%$ & {$[22]$} \\
\hline 9 & 15.508 & Carbidopa & 226.09909 & $11.53 \%$ & {$[25]$} \\
\hline 10 & 22.645 & N,N-Dimethylsphingosine & 309.30278 & $1.16 \%$ & {$[26]$} \\
\hline 11 & 22.696 & Caffeic acid & 180.04198 & $2.78 \%$ & {$[27]$} \\
\hline 12 & 23.754 & 7-Methylxanthine & 166.047 & $7.99 \%$ & {$[28]$} \\
\hline
\end{tabular}

Identification of the phytochemical constituents was done using LC-ESI-MS.

2.4. Experimental Animals. The animals (male BALB/c mice, 22-25 g) were bought from the Guangzhou University of Traditional Chinese Medicine (SYXK (YUE) 2018-0034, Guangzhou, China). All animal housing $\left(23 \pm 2{ }^{\circ} \mathrm{C}, 50 \pm 5 \%\right.$ humidity, $12 \mathrm{~h}$ light and dark cycle), handling, and feeding were supervised by the Animal Experimentation Ethics Committee at Guangzhou University of Traditional Chinese Medicine (Registration no. 20181224002).

After 7-day acclimation, all animals were assigned into 7 groups $(n=12)$ and received their respective treatments by oral gavage, as follows: control group and DSS group (distilled water, $0.1 \mathrm{~mL} / 10 \mathrm{~g}$ ), SASP-supplemented group (200 mg/kg), AZA-supplemented group $(13 \mathrm{mg} / \mathrm{kg})$, and EFH-supplemented groups (200, 400, and $800 \mathrm{mg} / \mathrm{kg}$, respectively). During the experiment, all groups except for the control group were exposed to 3\% DSS drinking freely for 10 days [14], and relative oral treatments were carried out once daily. The consumption of distilled water versus DSS solution between the groups was similar. The administrated doses of treatments were selected according to results from our pilot study and previous publications [15]. On the last day of the experiment, all the mice were sacrificed by carbon dioxide euthanasia; then, the colons were quickly removed and stored at $-80^{\circ} \mathrm{C}$ for further analysis.

2.5. Evaluation of Disease Activity Index (DAI). The DAI (body weight loss, stool character, and bloody feces) was evaluated by an observer daily, and the DAI score (calculated as [weight loss score + fecal trait score + hematochezia score]/3) was regarded as the standard scoring system [16]. After the mice were sacrificed, the colorectal lengths were measured. Portions of the distal colorectums were embedded and stained with hematoxylin and eosin ( $\mathrm{H} \& \mathrm{E})$. The histological scores were evaluated in a blinded manner [17].

2.6. Evaluation of the Levels of TNF- $\alpha, I F N-\gamma, I L-1 \beta, I L-6$, and MPO. The colorectal tissues were homogenized, and supernatants were collected by centrifugation. The levels of TNF$\alpha$, IFN- $\gamma$, IL- $1 \beta$, and IL- 6 were measured using ELISA kits at $450 \mathrm{~nm}$ according to the manufacturer's protocols, whereas MPO activity was determined with a myeloperoxidase assay kit at $460 \mathrm{~nm}$.

2.7. Quantitative Real-Time PCR Analysis. The total RNA was extracted from the colonic tissue with TRIzol reagent. After RNA concentration and purity control were completed, the high-quality RNA samples (RNA concentration $\geq 500$ $\mathrm{ng} / \mu \mathrm{L}$, OD260/OD280 $\geq 2.0$ ) were reverse transcribed to complementary DNA (cDNA) using FastKing Reverse Transcriptase Kit according to instructions from the supplier. The sequences of the primers used in this study are listed in Table 1. The QRT-PCR was performed with a ChamQ SYBR qPCR Master Mix (Vazyme Biotech Co., Ltd., Nanjing, China) and CFX Manager software (Bio-Rad Laboratories Inc.). The protocol was $95^{\circ} \mathrm{C}$ for $10 \mathrm{~min}$, followed by $95^{\circ} \mathrm{C}$ for $15 \mathrm{~s}$ and $60^{\circ} \mathrm{C}$ for $20 \mathrm{~s}$; this sequence was repeated for 40 cycles. The mRNA expression level (iNOS, COX-2, IL-4, IL-12, and IL-17) was calculated with the $2^{-\Delta \Delta \mathrm{Ct}}$ method relative to control gene GAPDH.

2.8. Western Blot Analysis. The total, cytoplasmic, and nuclear proteins were extracted using their corresponding extraction kits according to the manufacturer's statements. Briefly, the colonic tissues were homogenized with RIPA lysis buffer on ice. After incubation (20 min) and centrifugation $\left(14000 \times \mathrm{g}, 4^{\circ} \mathrm{C}, 10 \mathrm{~min}\right)$, the supernatant was collected as total protein for further analysis. The other samples were homogenized with PBS and centrifuged for $3 \mathrm{~min}(500 \times \mathrm{g}$, $4^{\circ} \mathrm{C}$ ). The sediment was mixed with Buffer A thoroughly for $30 \mathrm{~min}$ and then centrifuged $\left(12000 \times \mathrm{g}, 4^{\circ} \mathrm{C}, 10 \mathrm{~min}\right)$ again. The cytoplasmic protein was separated from the supernatant, whereas the nuclear protein was extracted from the sediment with Buffer B. After separated by SDS-PAGE, the proteins above were transferred onto PVDF membranes. Subsequently, the membranes were blocked in 5\% nonfat-dried milk and incubated with primary antibodies NF- $\kappa \mathrm{B}$ p-p65, NF- $\kappa \mathrm{B}$ p $65, \mathrm{I} \kappa \mathrm{B} \alpha, \mathrm{p}-\mathrm{I} \kappa \mathrm{B} \alpha, \mathrm{IKK} \alpha$, and $\mathrm{p}-\mathrm{IKK} \alpha$ (all $1: 1000$ dilution) overnight, followed by incubation with secondary antibody. The protein density was quantified by ImageJ software relative to $\beta$-actin or Histone $\mathrm{H} 3$. 


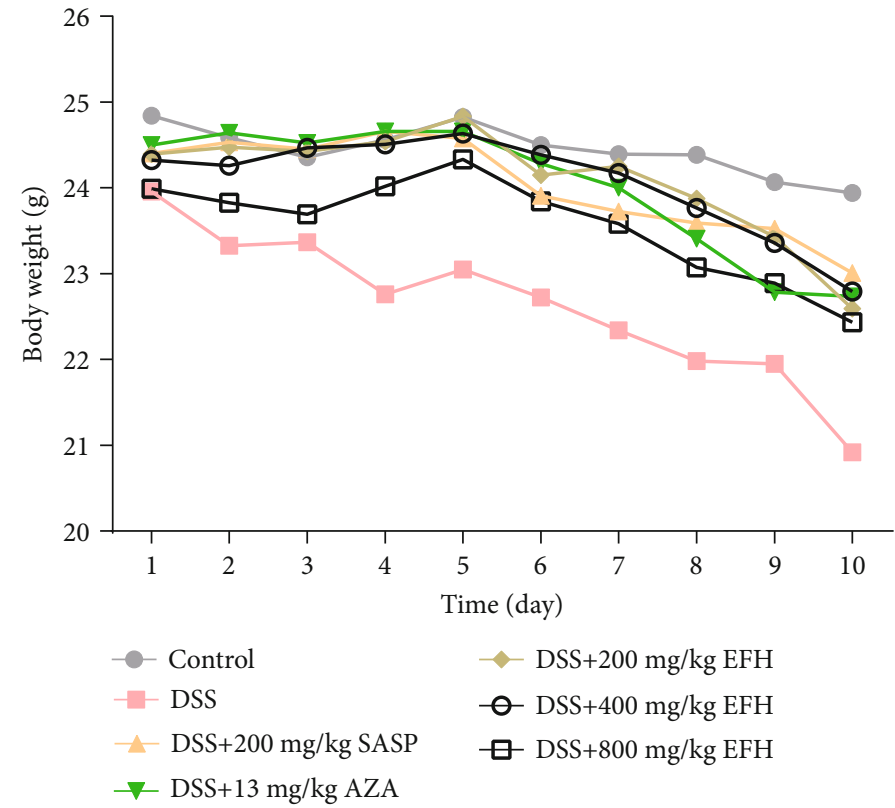

(a)

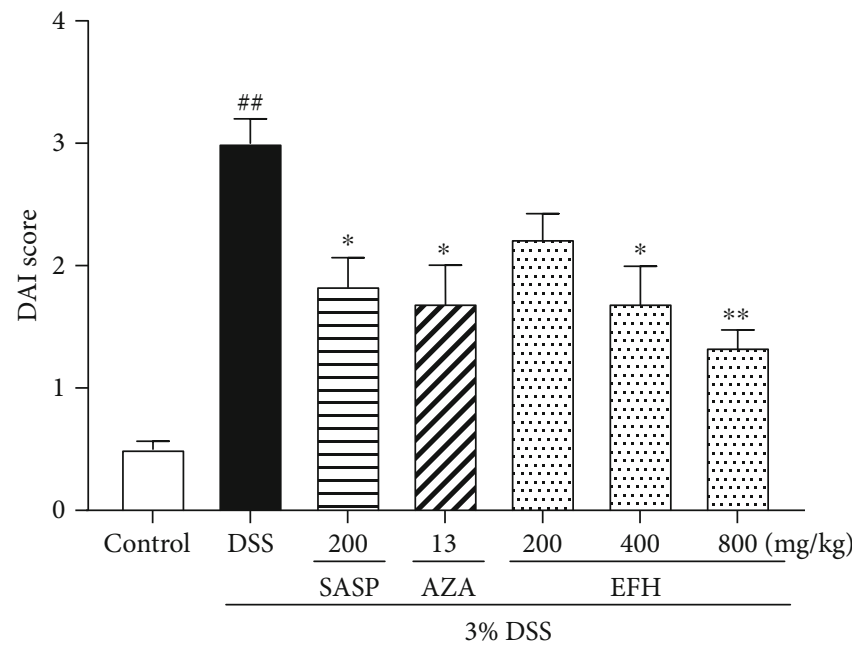

(b)

Figure 2: Continued. 

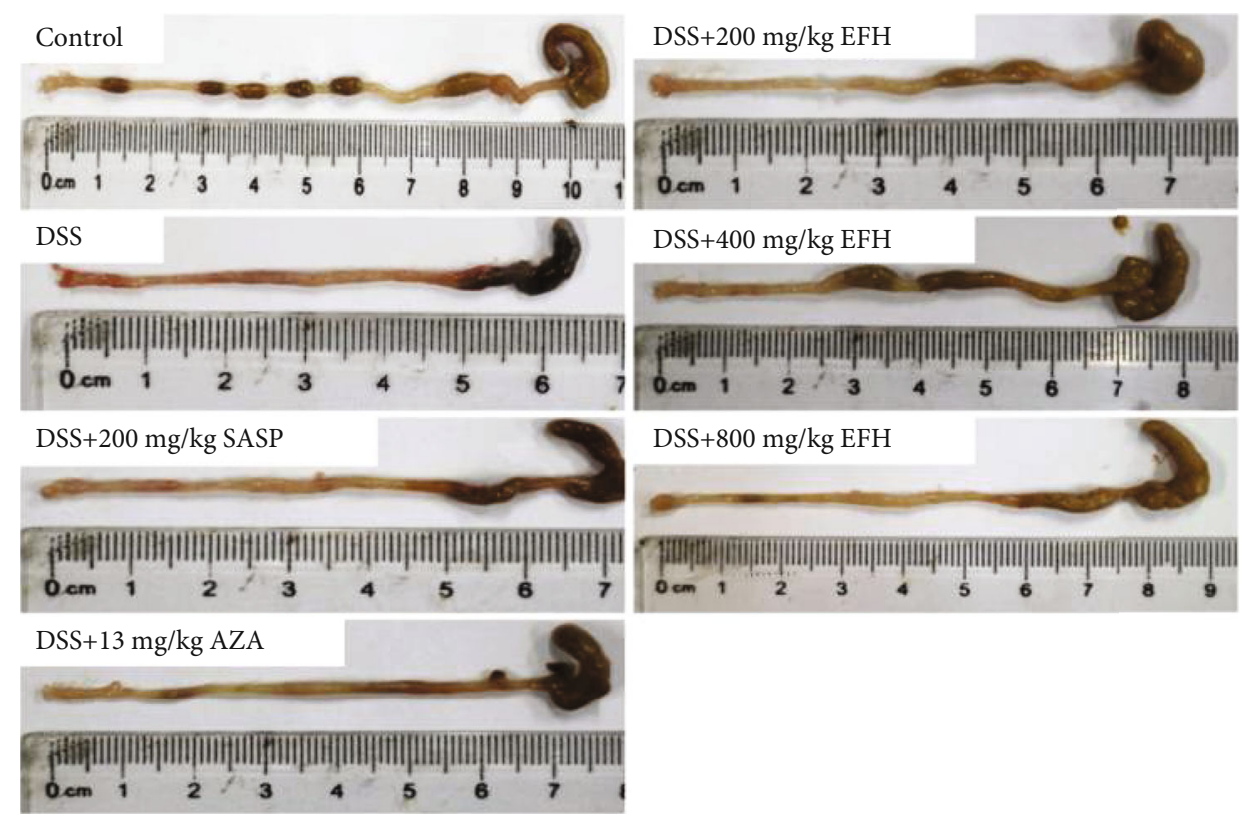

(c)

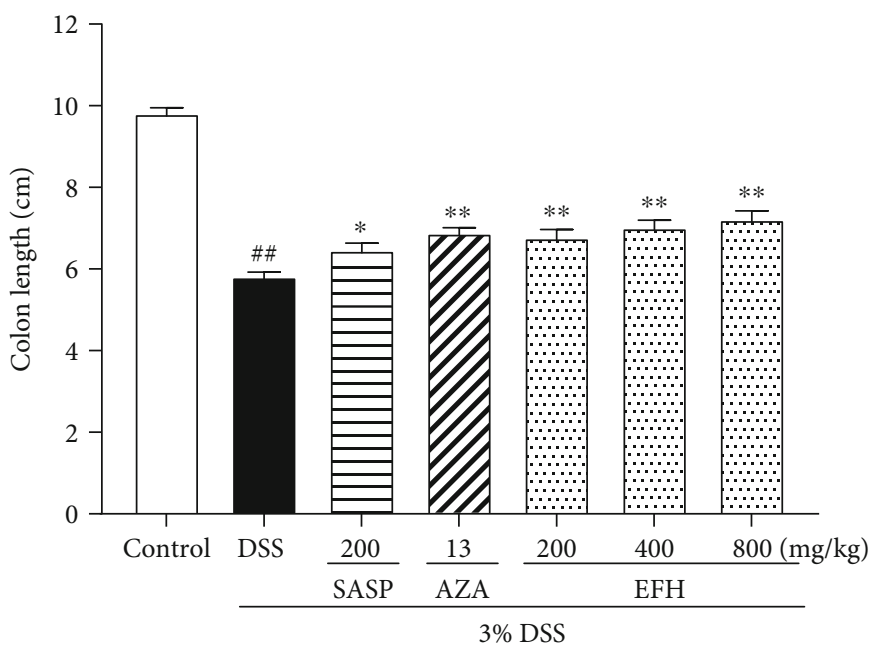

(d)

FIgURE 2: EFH ameliorated inflammatory indices of DSS-induced colitis. (a) The daily body weight changes from $1^{\text {st }}$ day to $10^{\text {th }}$ day. (b) The disease activity index (DAI) in mice. (c) The length of colons from a macroscopic perspective. (d) Quantitative measurement of colon length. Data are presented as the means \pm S.E.M. $(n=12) .{ }^{\# \#} p<0.01$ vs. the control group; $* p<0.05, * * p<0.01$ vs. the DSS group.

2.9. Gut Microbiota 16S rRNA Gene Sequencing and Analysis. Fecal genomic DNA was extracted using the DNA kit. The $16 \mathrm{~S}$ rDNA V4 region of the rRNA gene was amplified by PCR with the primer (515F: $5^{\prime}$-GTGCCAGCMGCCGCGGTAA- ${ }^{\prime}$ and 806R: $\quad 5^{\prime}$-GGACTACHVGGGTATCTAAT- $\left.3^{\prime}\right)$. Purified amplicons were sequence paired on an Illumina platform. The effective tags were clustered into operational taxonomic units (OTUs) of $\geq 97 \%$ similarity using USEARCH (v7.0.1090). On the basis of the relative abundance of OTUs, the microbial diversity and structural classification were further analyzed using programming language R (v3.1.1).

2.10. Statistical Analysis. All data were presented as the mean \pm standard error of mean (SEM) with Statistical Product and Service Solutions (SPSS) software (version 23.0).
Statistical analysis was carried out by one-way ANOVA followed by LSD test and Dunnett's test. A value of $p<0.05$ was considered statistically significant.

\section{Results}

3.1. The Phytochemical Constituent Analysis of EFH. The LCESI-MS analysis revealed that the high constituents were alkaloids and flavones in EFH. As shown in Figure 1 and Table 2, the chromatogram identified the phytochemical constituents in EFH as L-glutamic acid, triglycidyl glycerol, 2-naphthalenesulfonic acid, penicillic acid, syringic acid, puerarin, taxifolin, 4-hydroxycoumarin, carbidopa, N,Ndimethylsphingosine, caffeic acid, and 7-methylxanthine. These findings were consistent with previous publications. 


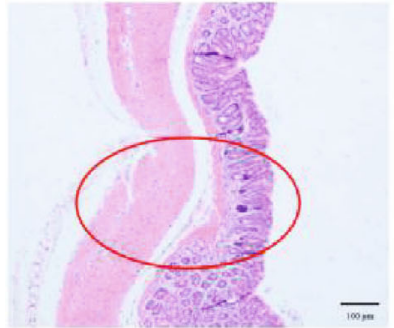

Control

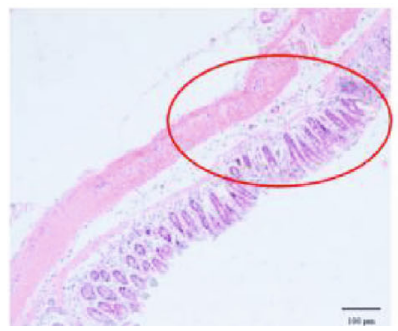

DSS+200 mg/kg EFH

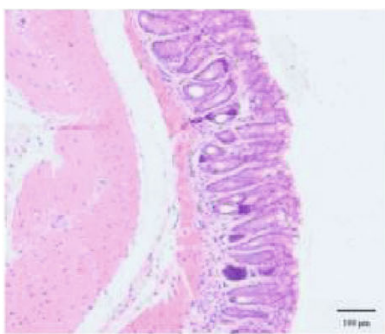

Control

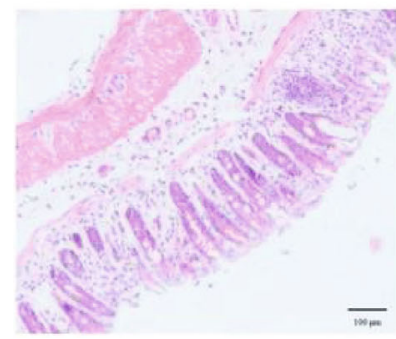

DSS+200 mg/kg EFH

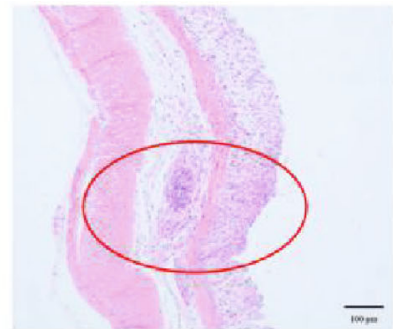

DSS

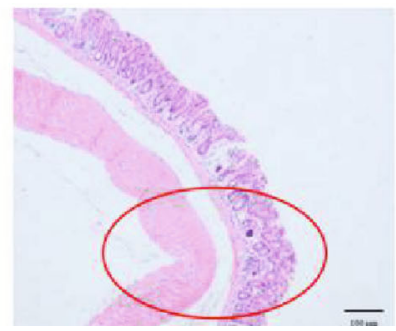

DSS $+400 \mathrm{mg} / \mathrm{kg} \mathrm{EFH}$

(a)

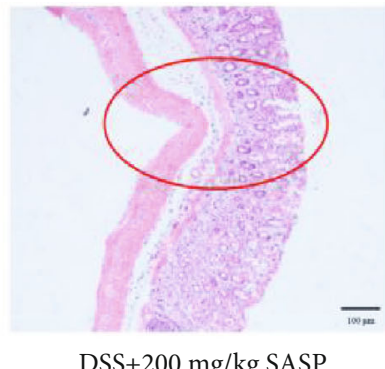

DSS+200 mg/kg SASP

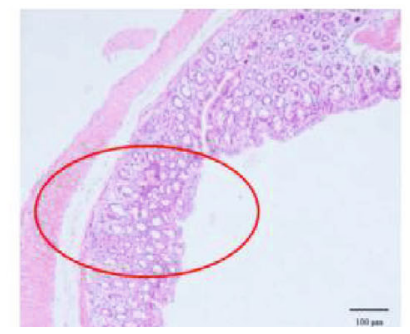

DSS+800 mg/kg EFH

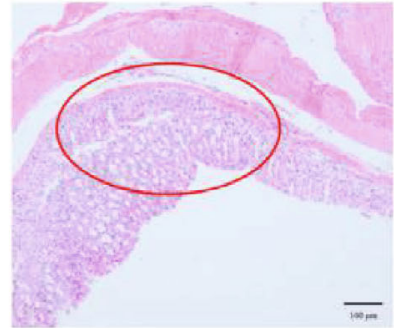

DSS+13 mg/kg AZA

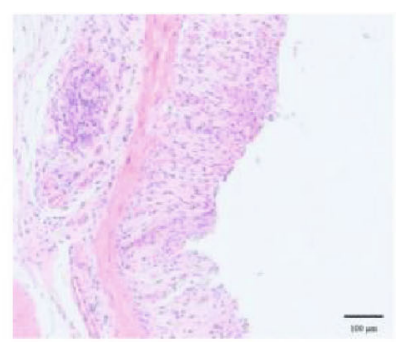

DSS

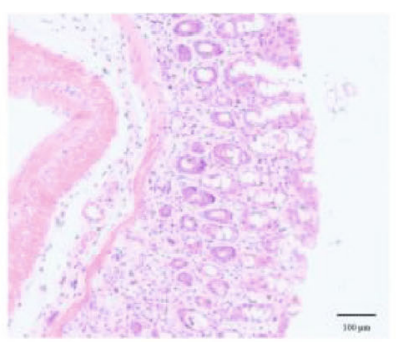

DSS+200 mg/kg SASP

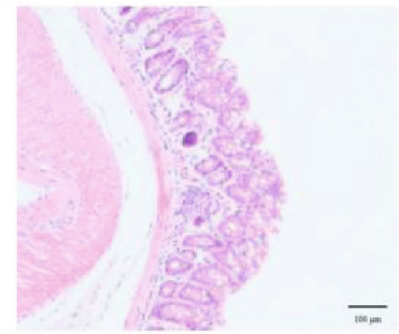

DSS+400 mg/kg EFH

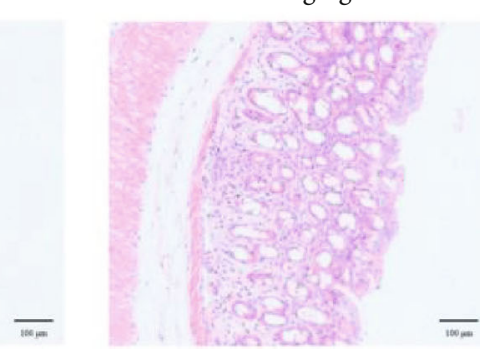

DSS+800 mg/kg EFH

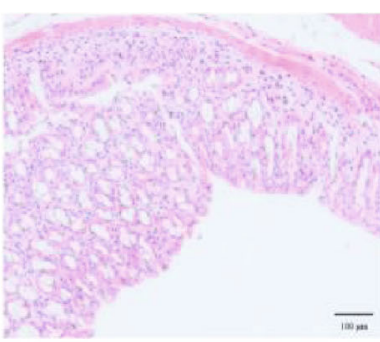

DSS+13 mg/kg AZA

(b)

Figure 3: Continued. 


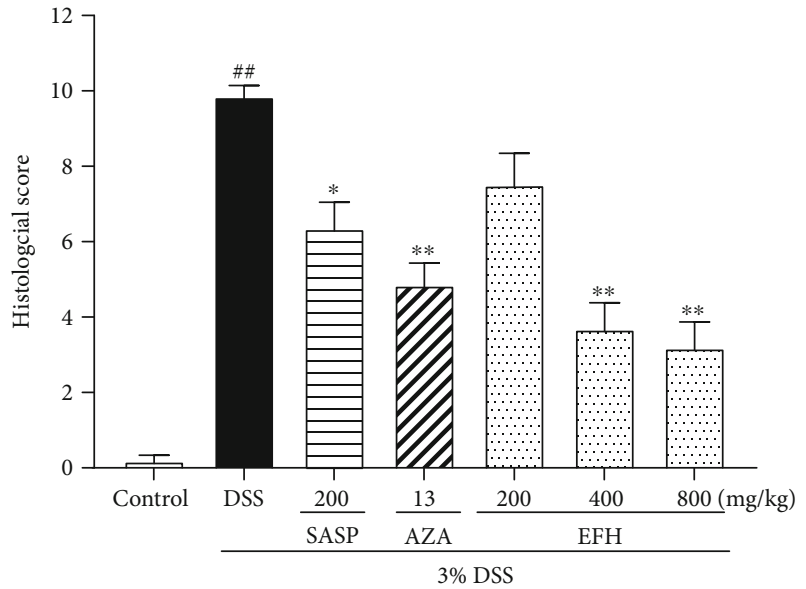

(c)

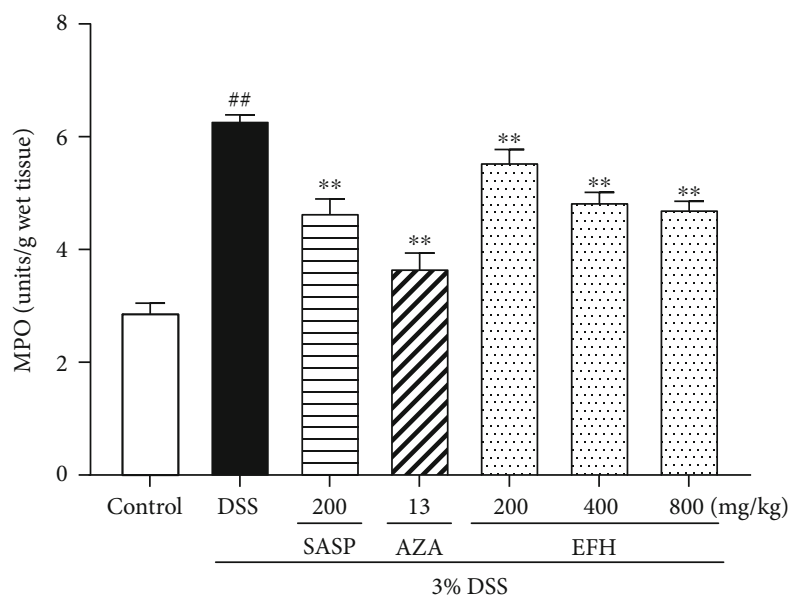

(d)

Figure 3: EFH suppressed inflammatory infiltration in DSS-induced colitis. H\&E staining of the formalin-fixed sections of colon (scale bars, $100 \mu \mathrm{m}$ ). (a) Magnification $\times 200$ (the parts in red circles were enlarged), (b) magnification $\times 400$, (c) histological scores, and (d) MPO activity of colon tissue in DSS-induced colitis mice. Data are presented as the means \pm S.E.M. $(n=10) .{ }^{\# \#} p<0.01$ vs. the control group; $* p<0.05$, $* * p<0.01$ vs. the DSS group.

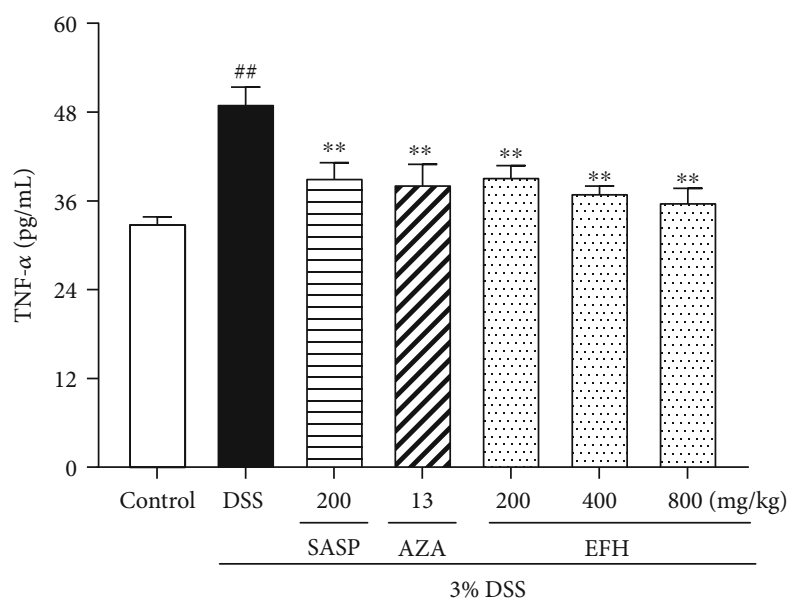

(a)

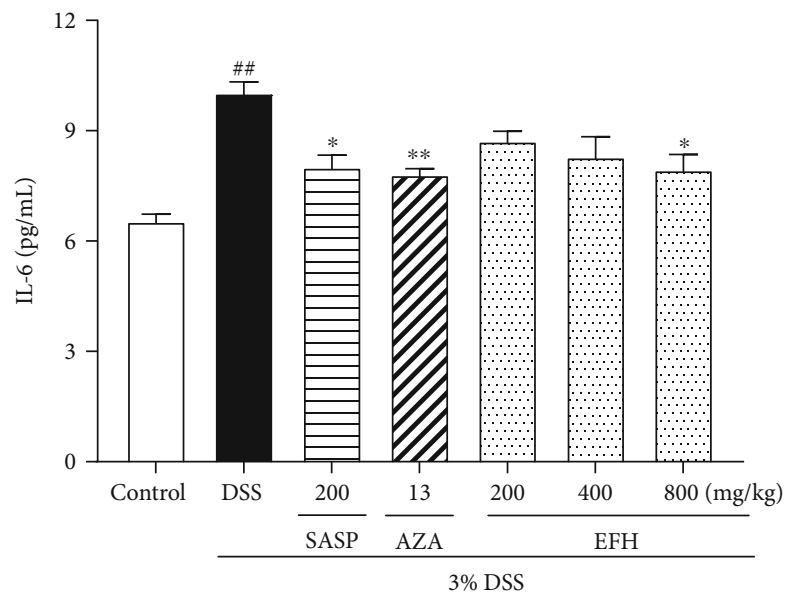

(c)

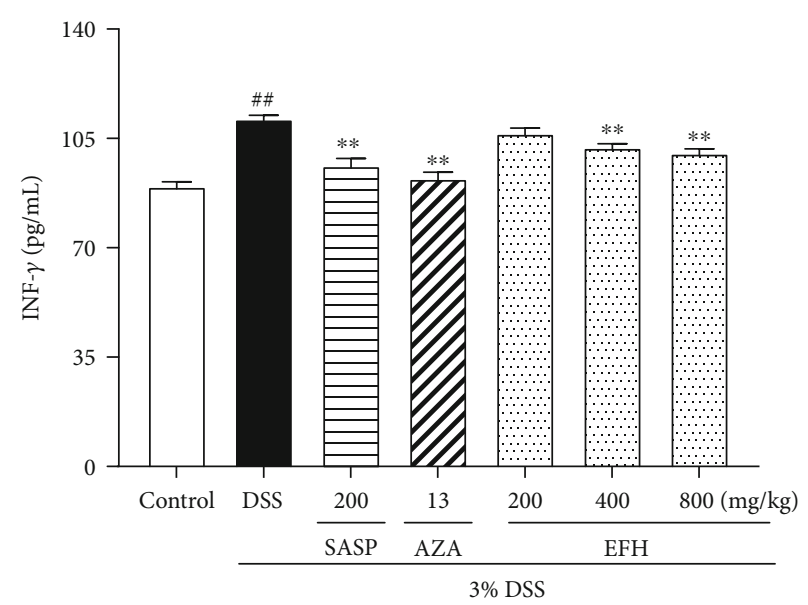

(b)

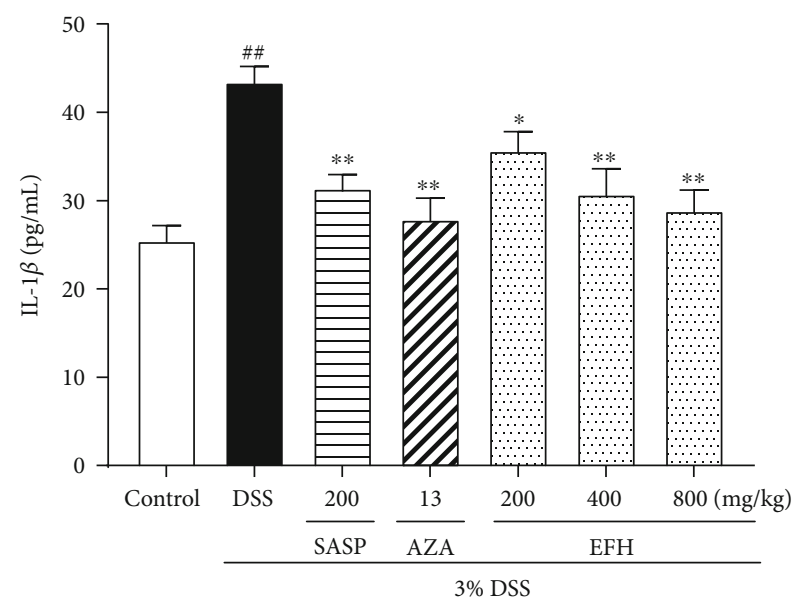

(d)

FIGURE 4: Effects of EFH on colonic level of proinflammatory cytokines TNF- $\alpha$ (a), IFN- $\gamma$ (b), IL-1 $\beta$ (c), and IL-6 (d) as determined by ELISA in DSS-induced colitis mice. Data are presented as the means \pm S.E.M. $(n=8) .{ }^{\# \#} p<0.01$ vs. the control group; $* p<0.05, * * p<0.01$ vs. the DSS group. 


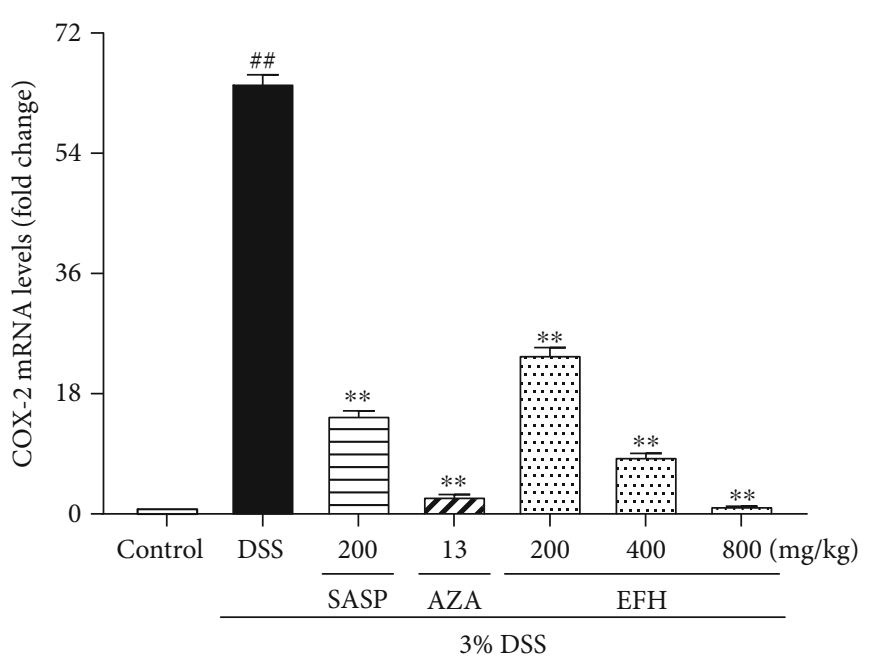

(a)

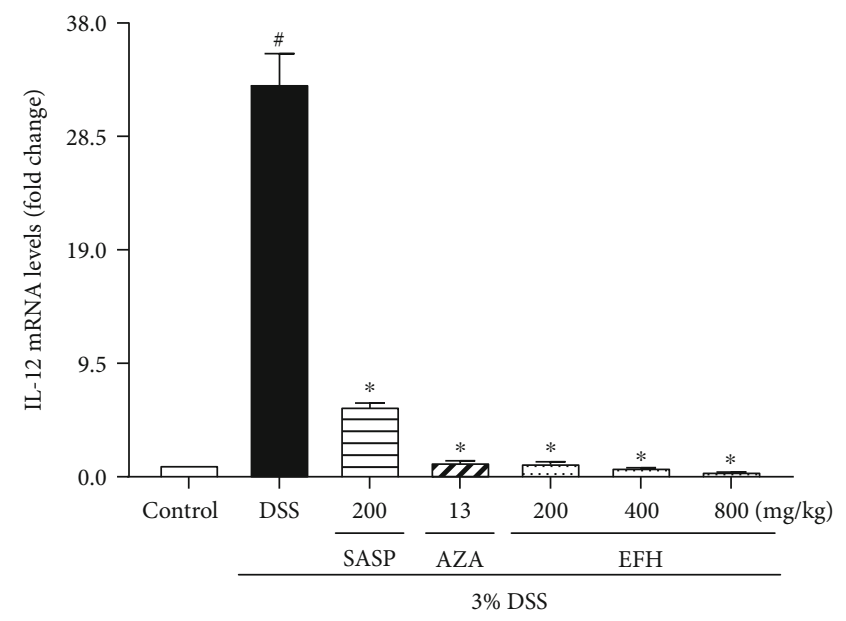

(c)

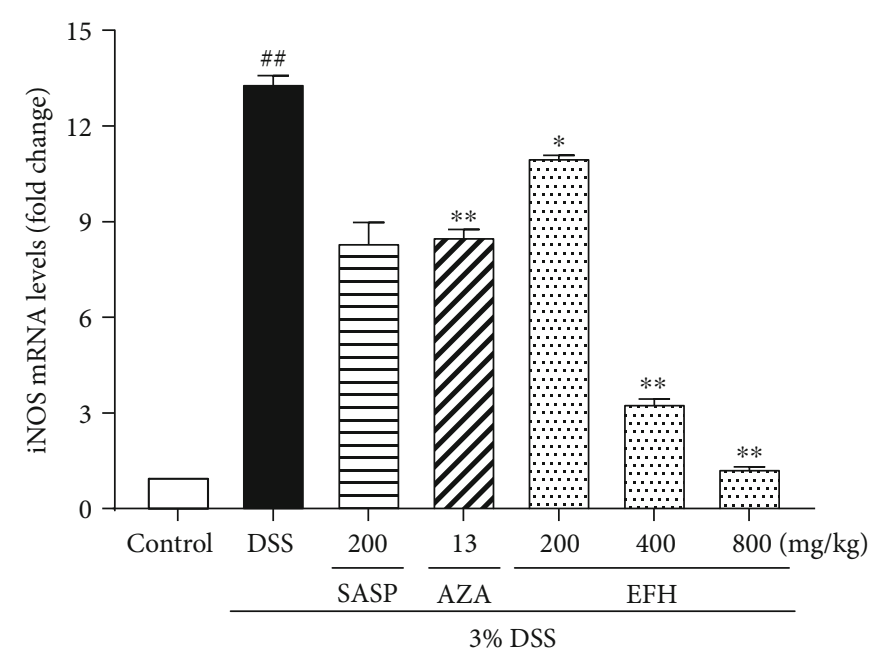

(b)

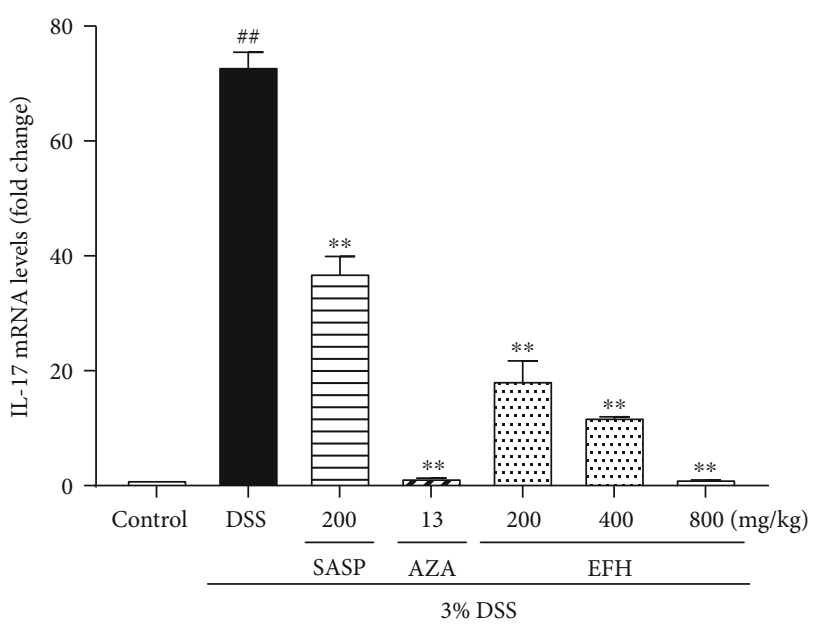

(d)

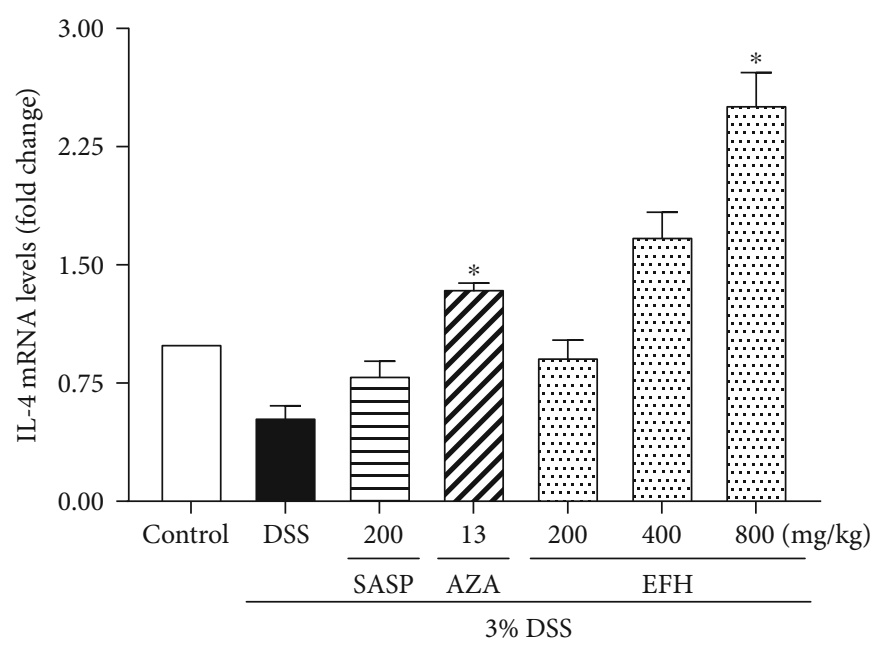

(e)

Figure 5: Effects of EFH on mRNA expression levels of COX-2 (a), iNOS (b), IL-12 (c), IL-17 (d), and IL-4 (e) in colorectums as determined by q-PCR in DSS-induced colitis mice. Data are presented as the means \pm S.E.M. $(n=3) .{ }^{\#} p<0.05,{ }^{\# \#} p<0.01$ vs. the control group; $* p<0.05$, $* * p<0.01$ vs. the DSS group. 


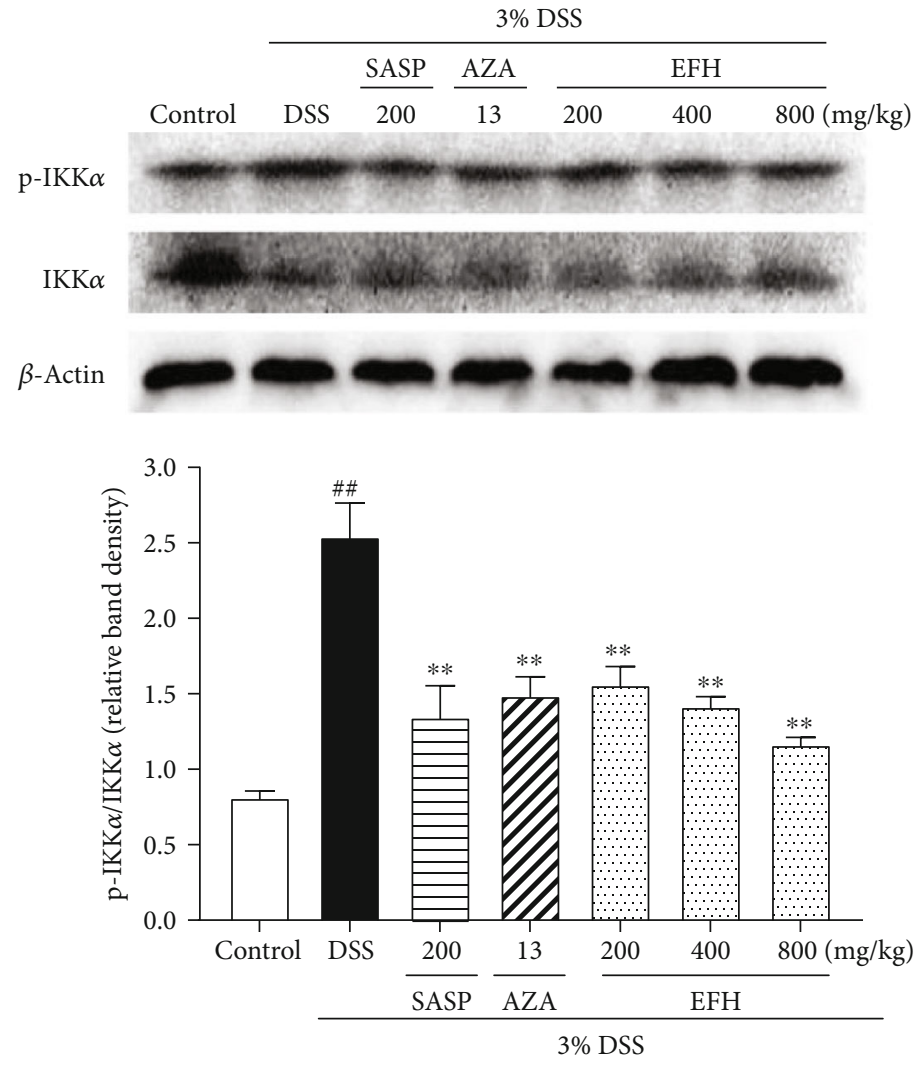

(a)
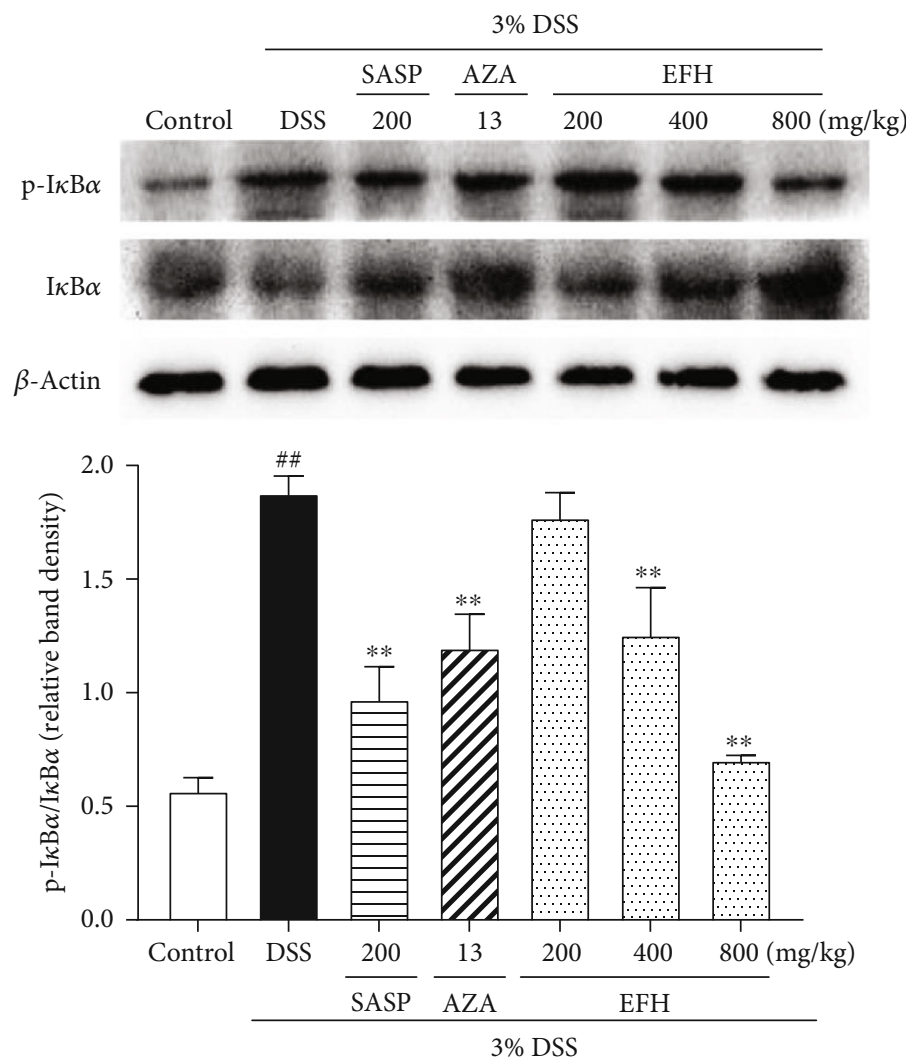

(b)

Figure 6: Continued. 


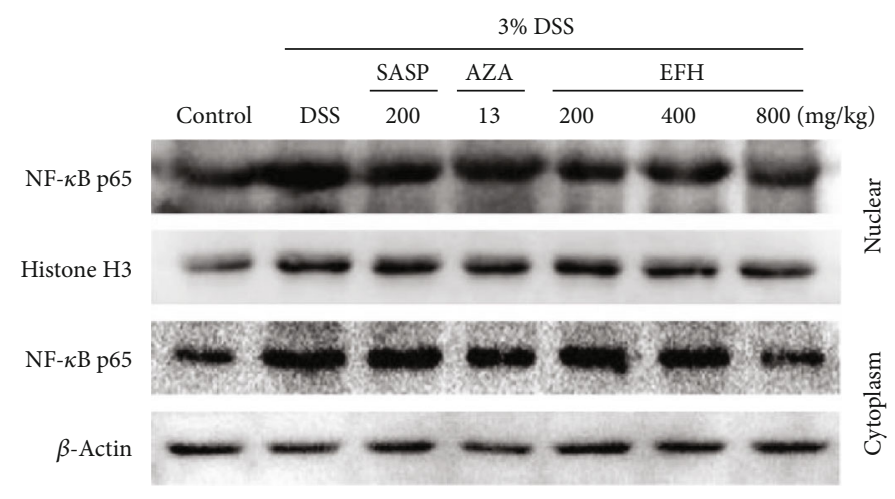

(c)

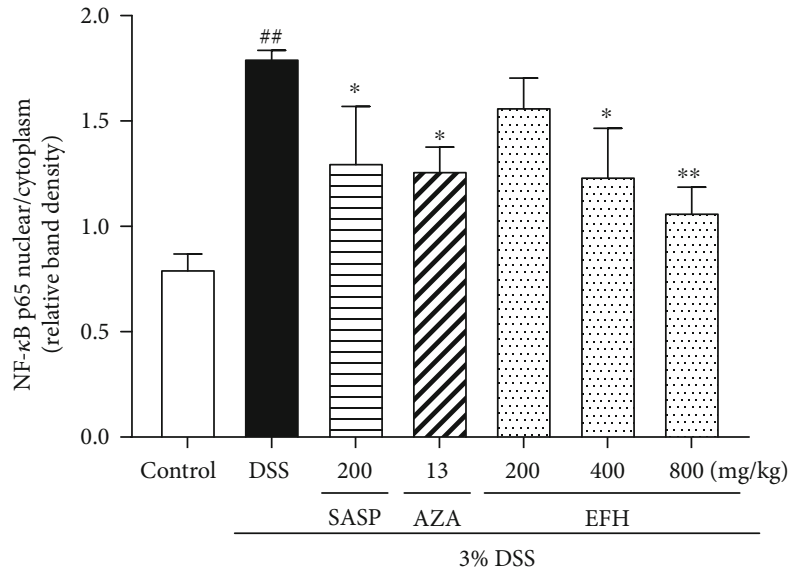

FIGURE 6: Effects of EFH on colonic expressions of the NF- $\kappa$ B pathway proteins determined by western blot in DSS-induced colitis mice. Densitometry analyses of western blots were determined by quantifying the protein levels of p-IKK $\alpha / \mathrm{IKK} \alpha(\mathrm{a}), \mathrm{p}-\mathrm{I} \kappa \mathrm{B} \alpha / \mathrm{I} \kappa \mathrm{B} \alpha(\mathrm{b})$, and $\mathrm{NF}$ $\kappa \mathrm{B}$ p65 nuclear/cytoplasm (c). Data are presented as the means \pm S.E.M. $(n=3) .{ }^{\# \#} p<0.01$ vs. the control group; $* p<0.05, * * p<0.01$ vs. the DSS group.

3.2. EFH Ameliorated Inflammatory Indices of DSS-Induced Colitis. The mice treated freely with 3\% DSS were used successfully to establish colitis. As shown in Figure 2(a), the body weight decrease on day 4 was significantly greater in the DSS group compared to the control group. However, the mice in the EFH groups exhibited a slight decrease in weight loss especially from day 5 . The results indicated that EFH treatment effectively alleviated DSS-induced weight loss.

Figure 2(b) showed that the DAI score of the DSS group dramatically increased $(p<0.01)$, whereas EFH treatment dose dependently decreased DAI scores even more than treatment with SASP or AZA. In addition, the DAI score was inversely associated with colon length, which was consistent with previous publication [29]. In Figures 2(c) and 2(d), the colon length in the DSS group was the shortest $(p<0.01)$, which was reversed by EFH in a dose-dependent manner (all $p<0.01)$.

3.3. EFH Suppressed Inflammatory Infiltration in DSSInduced Colitis. The results of H\&E staining and histopathological scores showed that the colonic tissue structures in the DSS group were destroyed compared to the control group (Figures 3(a) and 3(b)). Conversely, the groups treated with EFH reversed the changes in gut epithelial general morphology $(p<0.01)$. In addition, the MPO activity in DSS-treated mice remarkably increased compared to the control group $(p<0.01)$, suggesting growing infiltration of inflammatory cells. However, the groups treated with EFH, SASP, and AZA resulted in suppressing the MPO activity. Therefore, our results indicated that EFH significantly protected the intestinal epithelial structure.

3.4. EFH Suppressed the Levels of TNF- $\alpha, I F N-\gamma, I L-1 \beta$, and $I L-6$. To evaluate the anti-inflammatory effects of EFH, we measured the activity of proinflammatory cytokines by ELISA kits. As showed in Figure 4, the levels of all proinflammatory cytokines in the DSS group were much higher than those in the control group (all $p<0.01$ ). EFH treatment markedly lowered the expressions of TNF- $\alpha$, IFN- $\gamma$, IL- $1 \beta$, and IL-6, and the difference was significant in the mice treated with $800 \mathrm{mg} / \mathrm{kg}$ of EFH. Furthermore, the decrease observed in the mice receiving $800 \mathrm{mg} / \mathrm{kg}$ of $\mathrm{EFH}$ was close to that of the SASP and AZA groups.

3.5. EFH Decreased the mRNA Expressions of COX-2, iNOS, $I L-12, I L-17$, and $I L-4$. Many proinflammatory cytokines (COX-2, iNOS, IL-12, and IL-17) and an anti-inflammatory cytokine (IL-4) are involved in the initiation and development of intestinal inflammation. As shown in Figure 5, the levels of COX-2, iNOS, IL-12, and IL-17 in the DSS group were significantly higher than the level in the control group, and these high levels were reversed by EFH, SASP, or AZA. However, the anti-inflammatory cytokine IL-4 was upregulated in the EFH groups but was downregulated in the DSS group.

3.6. EFH Inhibited the Expressions of $N F-\kappa B$ Pathway. The $\mathrm{NF}-\kappa \mathrm{B}$ pathway plays an important role in the intestinal inflammation reaction, which is activated by $\mathrm{I} \kappa \mathrm{B} \alpha$ and IKK $\alpha$ phosphorylation [30]. The colonic tissue cell in the DSS group translocated NF- $\kappa \mathrm{B}$ p-p65 to the nucleus by phosphorylating $\mathrm{IKK} \alpha$ and $\mathrm{I} \kappa \mathrm{B} \alpha$ [31]. As shown in Figure 6, the activated NF- $\kappa$ B p65 $(p<0.05)$ and the phosphorylation of IKK $\alpha$ and $\mathrm{I} \kappa \mathrm{B} \alpha$ (all $p<0.01)$ in the DSS group were significantly higher than in the control group. On the contrary, treatment with EFH downregulated the expressions of NF$\kappa \mathrm{B}$ p-p65 p-IKK $\alpha$, and $\mathrm{p}-\mathrm{I} \kappa \mathrm{B} \alpha$, and these changes were similar to the effects of SASP and AZA.

3.7. EFH Altered Microbial Structure and Diversity of Gut Microbiota in Mice. Changes in the intestinal microenvironment have been reported in UC [32]. The operational taxonomic units (OTU) rank curve represents the number of observed OTUs in all samples. As showed in Figure 7(b), the DSS group had significantly fewer OTUs than other groups $(p<0.01)$, but the number of OTUs was enriched by $\mathrm{EFH}$ treatment. Additionally, the plateaued rarefaction 


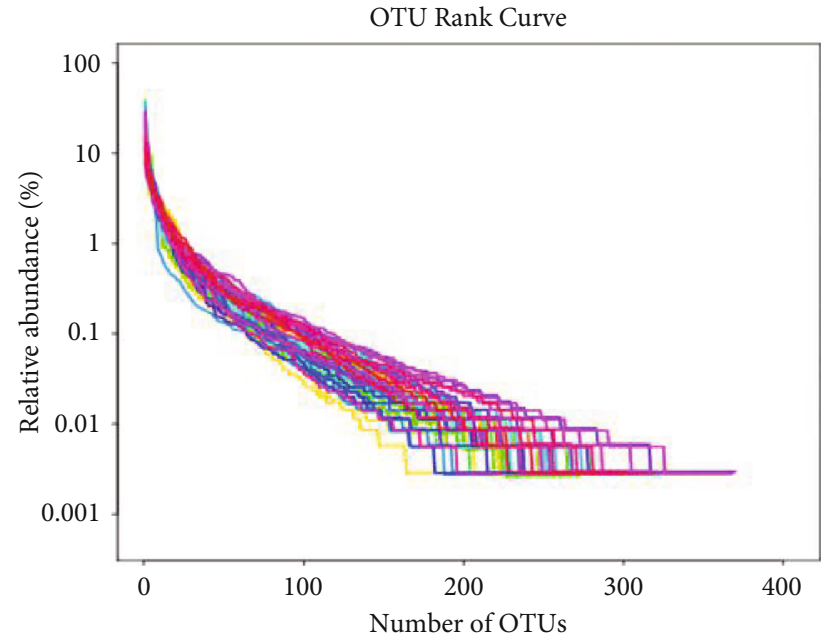

$\begin{array}{llr}\text { AZA1 } & \text { EL4 } & \text { M7 } \\ \text { AZA2 } & \text { EL5 } & \text { M8 } \\ \text { AZA3 } & \text { EL7 } & \text { N1 } \\ \text { AZA4 } & \text { EL8 } & \text { N3 } \\ \text { AZA5 } & \text { EM1 } & \text { N4 } \\ \text { AZA6 } & \text { EM2 } & \text { N5 } \\ \text { AZA7 } & \text { EM3 } & \text { N6 } \\ \text { AZA8 } & \text { EM4 } & \text { N7 } \\ \text { EH1 } & \text { EM5 } & \text { N8 } \\ \text { EH2 } & \text { EM6 } & \text { SP1 } \\ \text { EH3 } & \text { EM7 } & \text { SP2 } \\ \text { EH4 } & \text { EM8 } & \text { SP3 } \\ \text { EH5 } & \text { M1 } & \text { SP4 } \\ \text { EH6 } & \text { M2 } & \text { SP5 } \\ \text { EH7 } & \text { M3 } & \text { SP6 } \\ \text { EH8 } & \text { M4 } & \text { SP7 } \\ \text { EL1 } & \text { M5 } & \text { SP8 } \\ \text { EL2 } & \text { M6 } & \\ \text { EL3 } & & \end{array}$

(a)

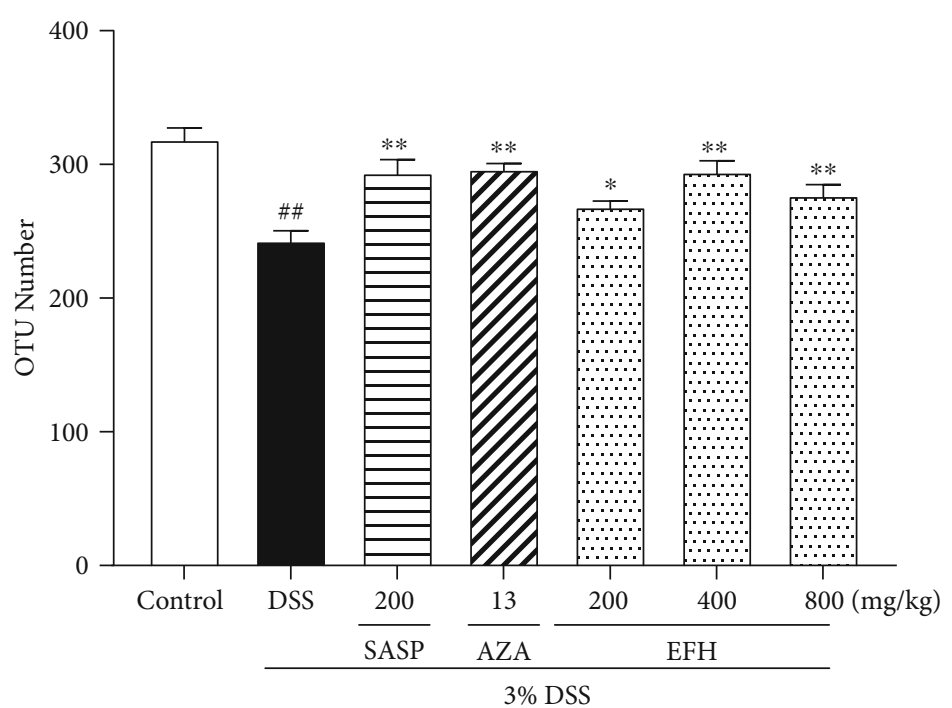

(b)

Figure 7: Continued. 

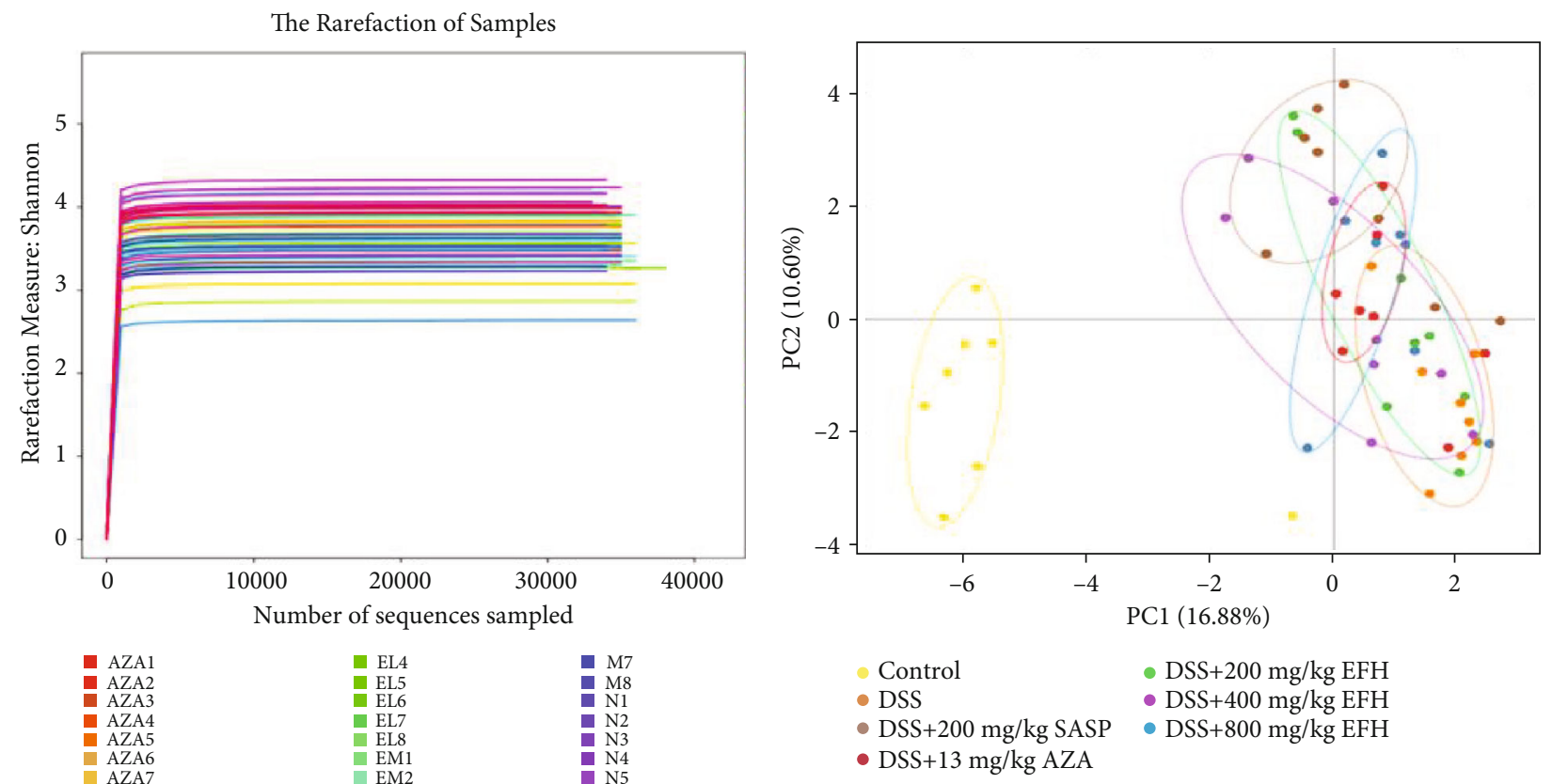

$\begin{array}{llr}\text { AZA1 } & \text { EL4 } & \text { M7 } \\ \text { AZA2 } & \text { EL5 } & \text { M8 } \\ \text { AZA3 } & \text { EL7 } & \text { N1 } \\ \text { AZA4 } & \text { EL8 } & \text { N2 } \\ \text { AZA5 } & \text { EM1 } & \text { N3 } \\ \text { AZA6 } & \text { EM2 } & \text { N4 } \\ \text { AZA7 } & \text { EM3 } & \text { N6 } \\ \text { AZA8 } & \text { EM4 } & \text { N7 } \\ \text { EH1 } & \text { EM5 } & \text { N8 } \\ \text { EH2 } & \text { EM6 } & \text { SP1 } \\ \text { EH3 } & \text { EM7 } & \text { SP2 } \\ \text { EH4 } & \text { EM8 } & \text { SP3 } \\ \text { EH5 } & \text { M1 } & \text { SP4 } \\ \text { EH6 } & \text { M2 } & \text { SP5 } \\ \text { EH7 } & \text { M3 } & \text { SP6 } \\ \text { EH8 } & \text { M4 } & \text { SP7 } \\ \text { EL1 } & \text { M5 } & \text { SP8 } \\ \text { EL2 } & \text { M6 } & \end{array}$

(c)

(d)

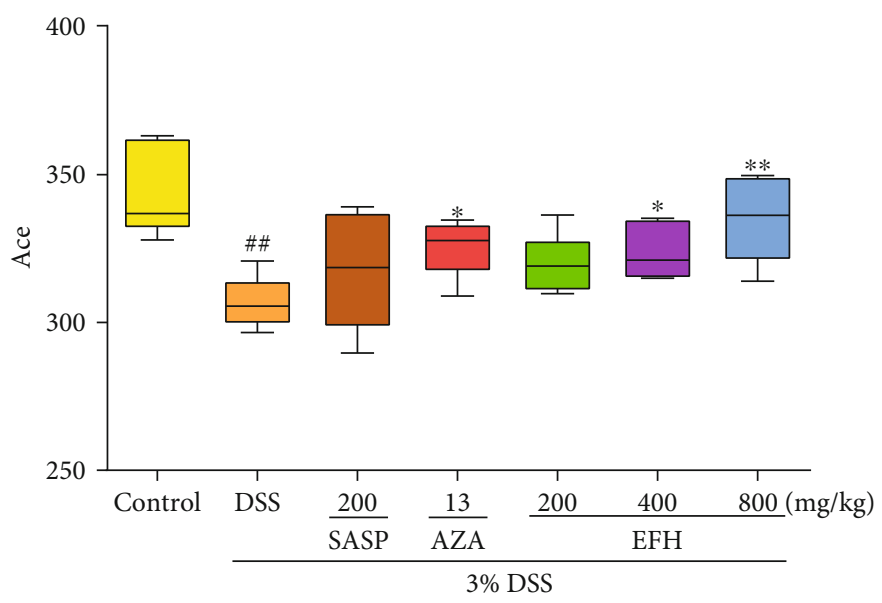

(e)

Figure 7: Continued. 


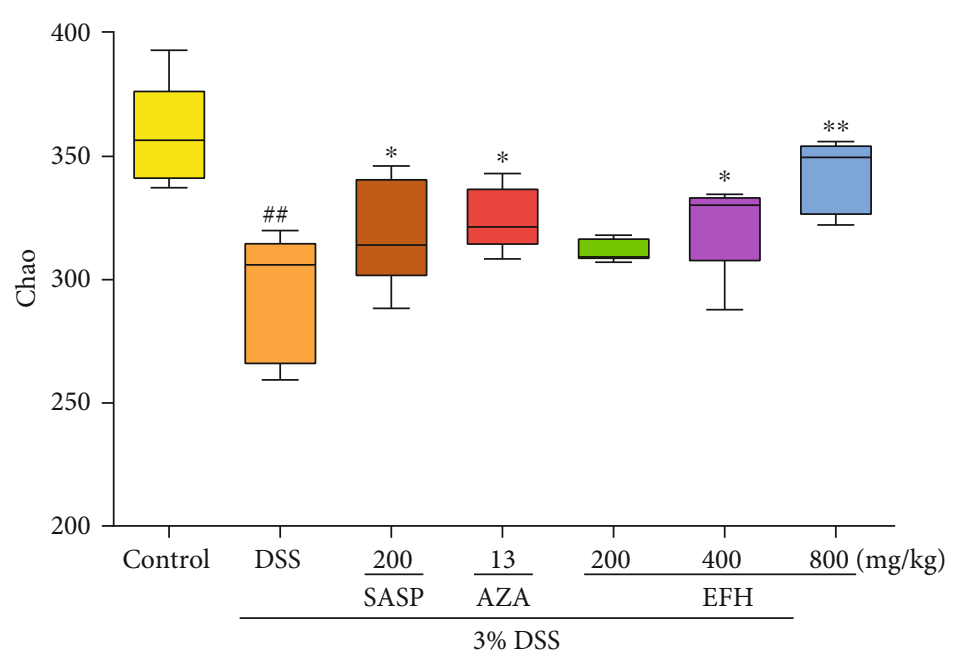

(f)

Figure 7: Effects of EFH on the overall structure and microbial diversity of the gut microbiota in DSS-induced colitis mice. (a) OTU rank curve. (b) The histogram of OTU number. (c) Shannon's rarefaction curve of samples. (d) Multiple sample PCA analysis. (e) Ace and (f) Chao of alpha diversity analysis. Data are presented as the means \pm S.E.M. $(n=3)$. ${ }^{\# \#} p<0.01$ vs. the control group; $* p<0.05, * * p<0.01$ vs. the DSS group.

curve in Figure 7(c) indicated that the sequencing depth of all samples had basically finished. The principal component analysis (PCA) revealed that the microbial community structure of the DSS group deviated from that of control group, whereas EFH treatment partially mitigated the shift (Figure $7(\mathrm{~d})$ ). According to alpha diversity analysis (Figures 8(e) and 8(f)), a significant reduction in microbial diversity was observed in the DSS group, but higher diversity was recorded in the EFH groups.

3.8. EFH Regulated Gut Microbiota Structure in DSS-Induced Colitis Mice. The histograms in Figure 8 revealed the gut microbiota community structure and the marked percentage showed the relative abundance at all levels. The results indicated that DSS decreased the relative abundance of Bacteroidetes and Firmicutes by $2.4 \%$ and $3.2 \%$, respectively, compared with the control group, whereas DSS increased the levels of Proteobacteria by $6.8 \%$. However, EFH reversed the proportions of all 3 phyla to beyond those of the control group. At the class level (Figure 8(b)), the levels of Bacteroidia and Clostridia dropped in the DSS group, which was reversed by EFH treatment. In addition, $\varepsilon$-Proteobacteria and $\delta$-proteobacteria related to IBD pathogenesis [33] were found at high levels in the DSS group.

As shown in Figure 8(c), Bacteroidales, Clostridiales, Campylobacterales, and Desulfovibrionales were the most represented of the 12 bacterial orders. Among them, Campylobacterales was identified at a relatively high level in the DSS group but was detected only slightly in the EFH groups. Most of the 16 families observed were related to the communities observed at the phylum and class levels. Lachnospiraceae and S24-7 were the main intestinal floras in the control group, and their levels were reduced by DSS treatment. After EFH treatment, the levels of these bacteria recovered to levels similar to the control group.
As shown in Figure 8(e), sequencing data identified 18 genera of intestinal microflora. The relative abundance of Odoribacter was significantly downregulated by DSS treatment, but EFH treatment reversed the alteration. In addition, EFH reduced the levels of Helicobacter, Ruminococcus, and Paraprevotella.

\section{Discussion}

In this investigation, we proved for the first time the effectiveness of EFH to treat DSS-induced colitis by improving the balance of the gut microbiota and inhibiting the NF- $\kappa \mathrm{B}$ pathway. DSS-induced colitis is similar to UC in human [34], so we extrapolated the anti-UC mechanism of EFH based on the basis of results in mice with DSS-induced colitis.

Our LC-ESI-MS chromatogram results showed that EFH is a complex mixture of chemical compositions. The main effective components of EFH were flavonoids, predominantly taxifolin and puerarin. Taxifolin exerts its diverse therapeutic benefits in inflammation-related diseases via the inhibition of the NF- $\kappa \mathrm{B}$ pathway [35]. Puerarin is associated with changes in the gut microbiota and inhibits the NF- $\kappa \mathrm{B}$ pathway to relieve the inflammatory response [36]. Therefore, we speculated that the observed anti-inflammatory effects of $\mathrm{EFH}$ might be associated with flavonoids. However, more research is needed to clarify how the biologically active ingredients of EFH act on DSS-induced colitis.

The DAI score, colon length, and histological changes are some of the markers used to assess for inflammation in $\mathrm{BALB} / \mathrm{c}$ mice with DSS-induced colitis [29]. Our results showed that mice treated with EFH maintained body weight and had significantly decreased DAI scores and histological scores. In addition, MPO activity was higher in the DSS group but was lowered with EFH treatment, suggesting that EFH attenuated DSS-induced massive inflammatory 


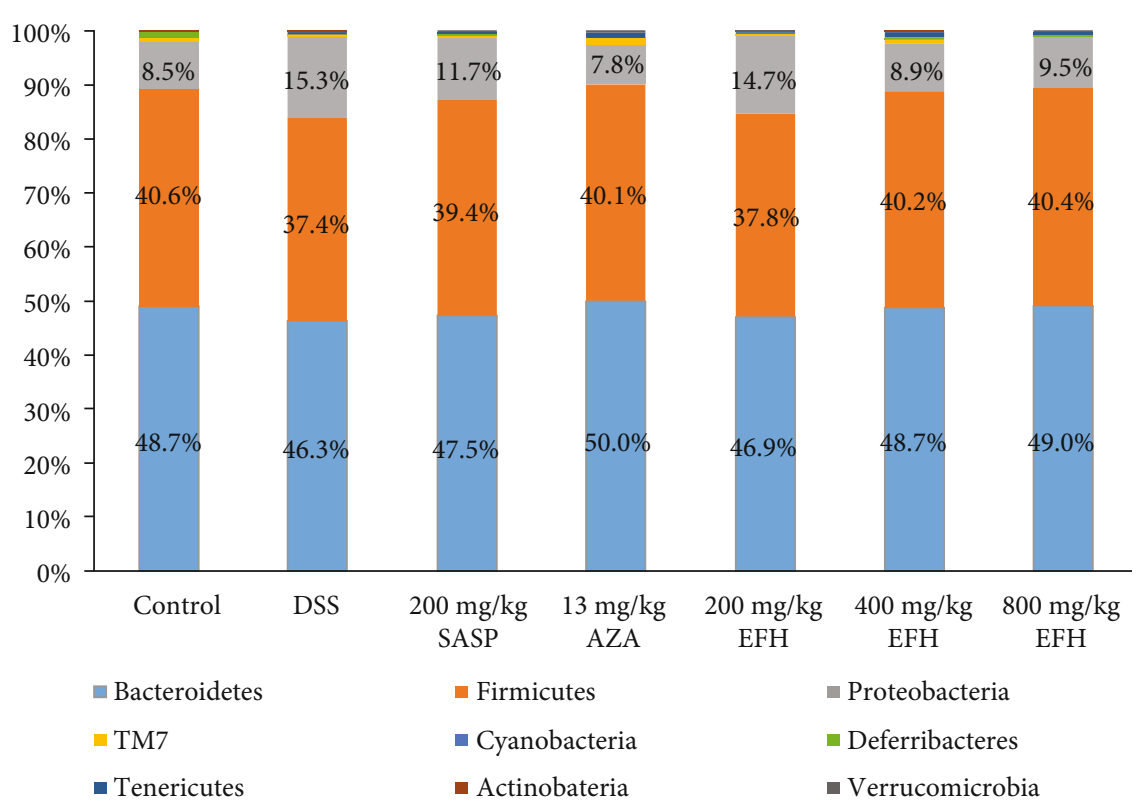

(a)

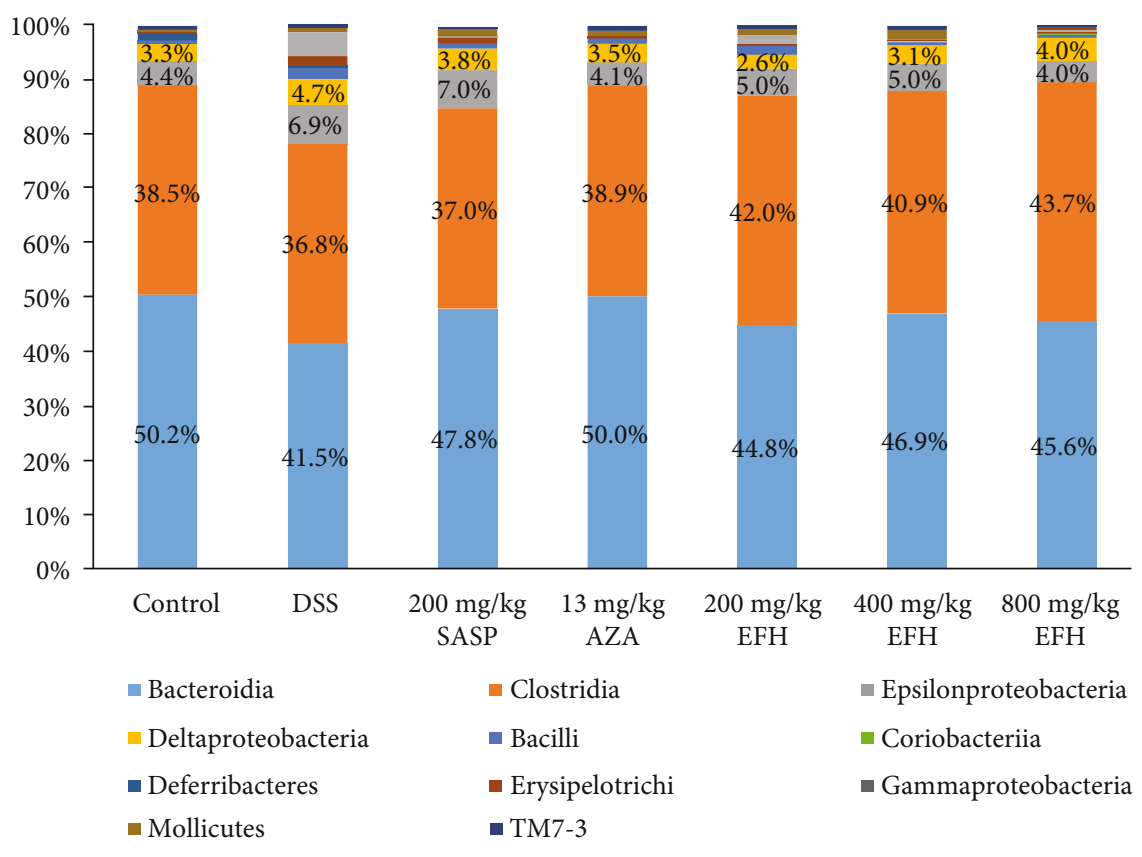

(b)

Figure 8: Continued. 


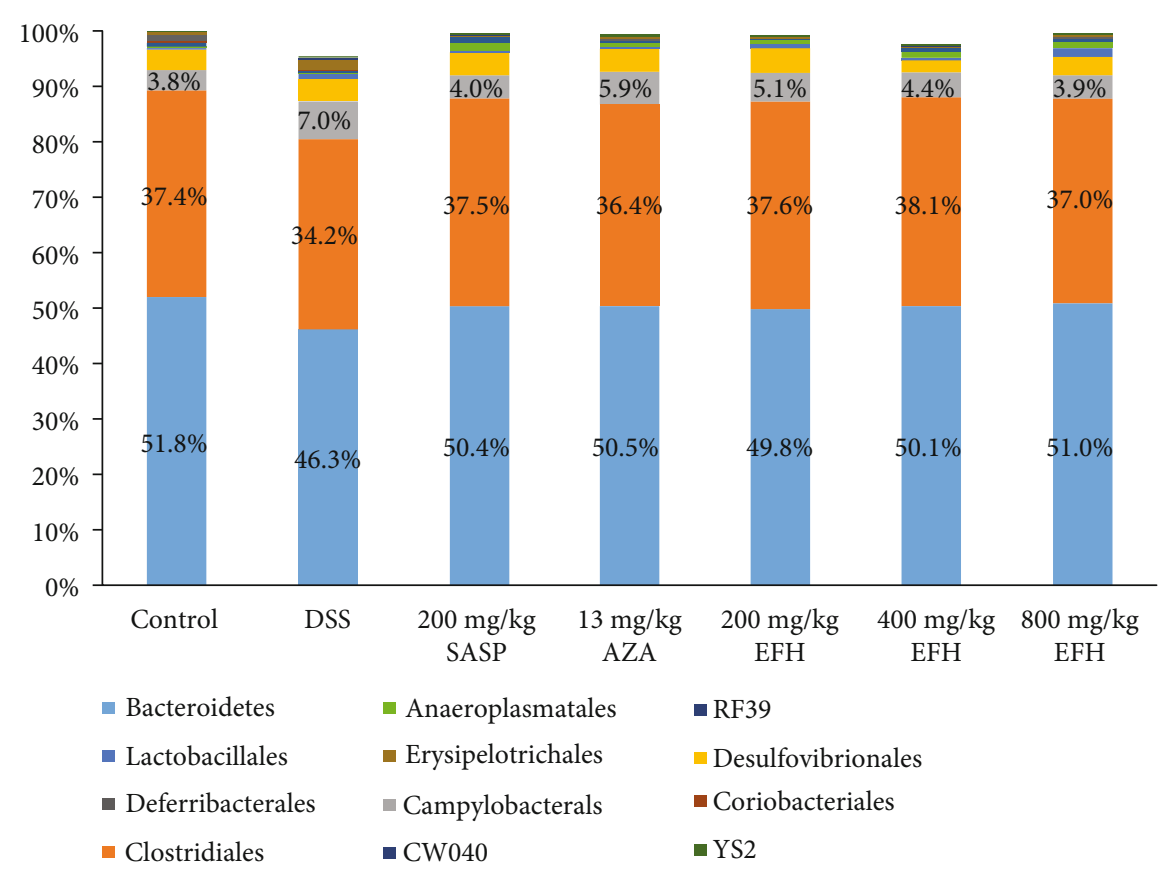

(c)

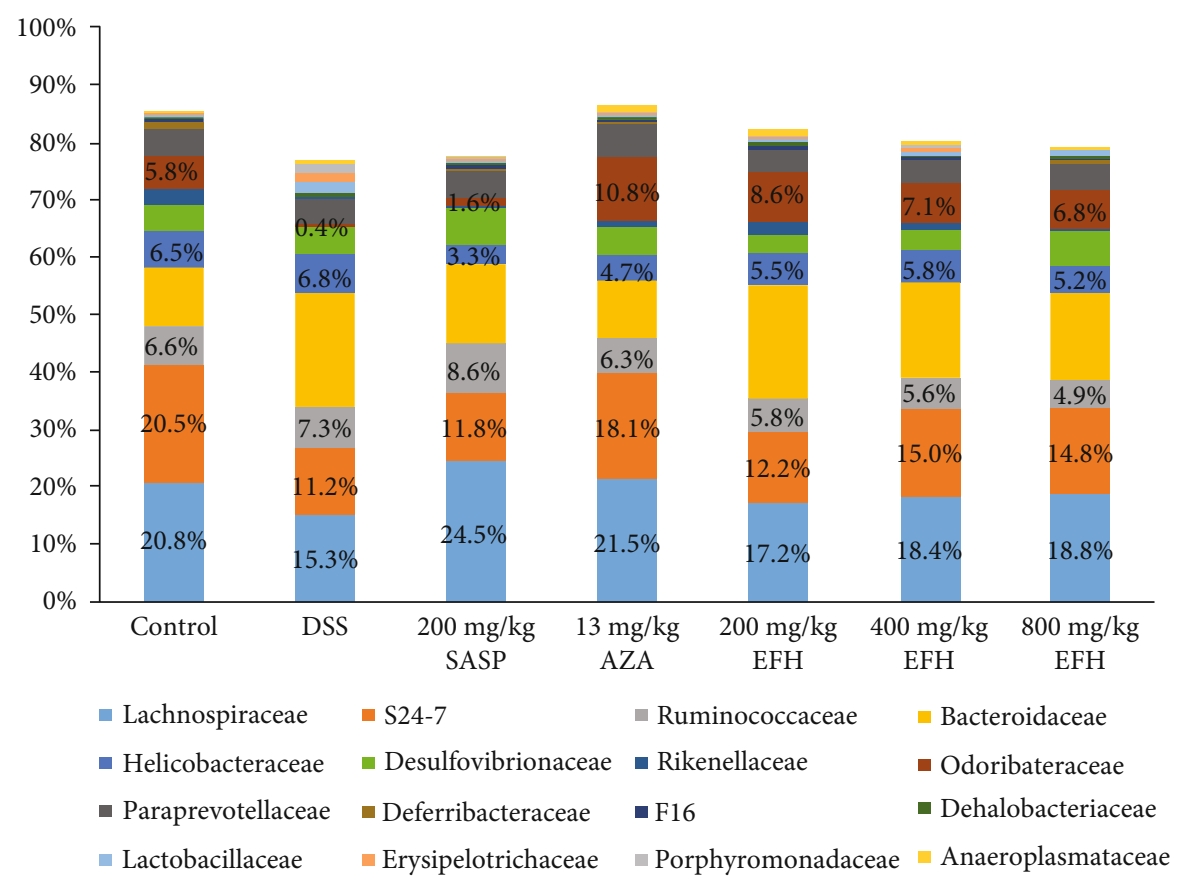

(d)

Figure 8: Continued. 


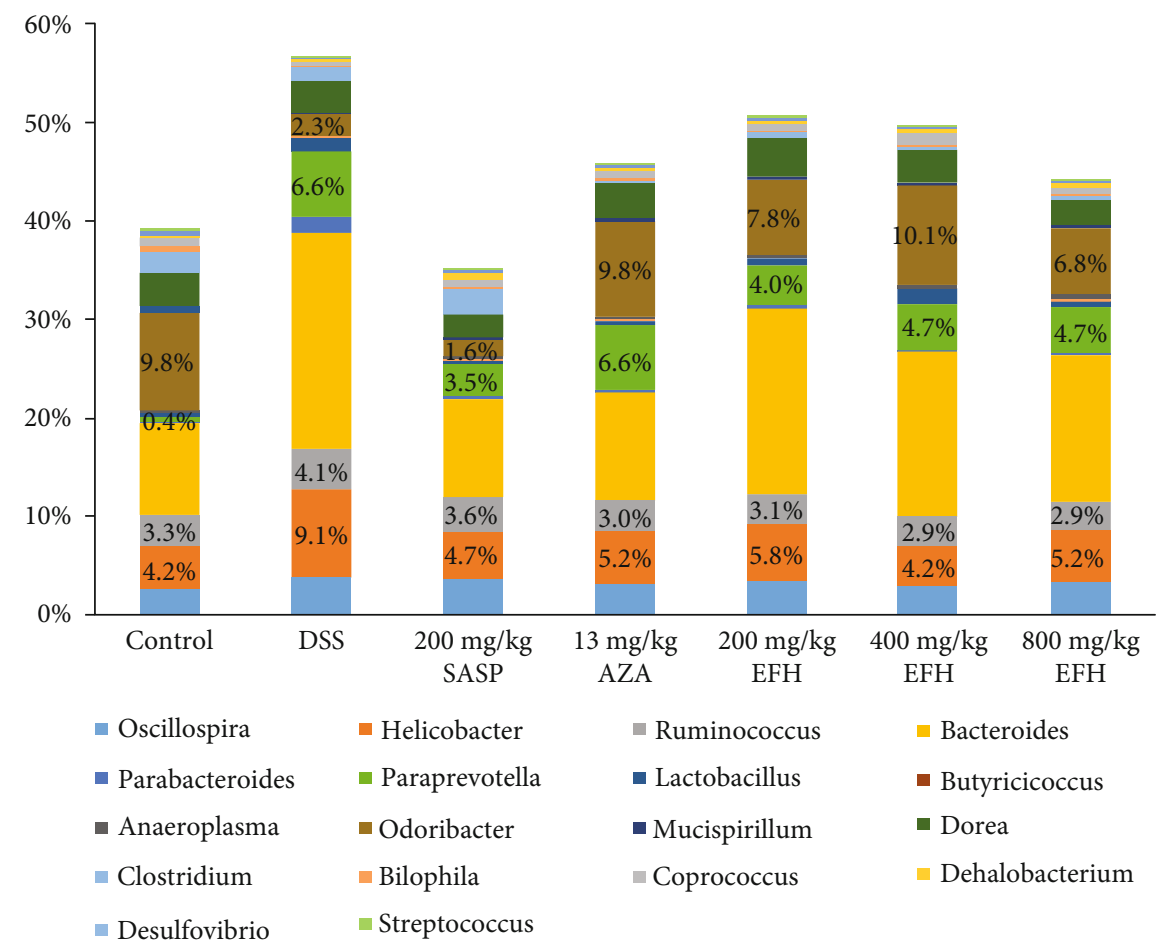

(e)

FIGURE 8: Effects of HQD on structural segregation of the gut microbiota in DSS-induced colitis mice. (a) Phylum, (b) class, (c) order, (d) family, and (e) genus $(n=5)$.

infiltration and disruption of mucosal structures [37]. These results suggested that EFH exerted noticeably protective effects against DSS-induced colitis to alleviate colonic inflammation.

In recent years, the gut microbiota has received great attention, and many studies have explored the changes of the intestinal flora occurring with intestinal diseases, including IBD and CRC [38]. In our study, DSS reduced the microbial diversity of the gut microbiota in mice with colitis [39]. In contrast, EFH improved the bacterial diversity and kept the balance between the population of beneficial bacteria and pathogenic bacteria. For example, Helicobacter is associated with chronic gastritis and peptic ulcer diseases [40], and we found that EFH reduced the relative increase of Helicobacter caused by DSS. Remarkably, EFH treatment increased the richness of Odoribacter compared with DSS treatment, and Odoribacter may reduce SCFA production to relieve UC [41]. These results also suggested that EFH regulated the gut microbiota to keep the balance of intestinal homeostasis in DSS-induced colitis. However, the relationship between the gut microbiota and colitis must be investigated further.

Dysbiosis of the gut microbiota is associated with the destruction of intestinal barrier and signals to epithelial cells to trigger inflammatory responses [42]. The dysbiosis of cytokines and gut bacteria might be controlled by the NF$\kappa \mathrm{B}$ pathway [43]. The phosphorylation of p65 was thought to enhance its entrance into the nucleus, so we measured the expression level of the NF- $\kappa \mathrm{B}$ p 65 nuclear/cytoplasm ratio. According to our results, EFH relieved DSS-induced inflammatory infiltration in epithelial cells and then suppressed the NF- $\kappa \mathrm{B}$ pathway through downregulation of the phosphorylated proteins IKK $\alpha, \mathrm{I} \kappa \mathrm{B} \alpha$, and NF- $\kappa \mathrm{B}$ p 65 . The activated NF- $\kappa \mathrm{B}$ pathway can be associated with the high expression of cytokines. Therefore, proinflammatory cytokines (TNF- $\alpha$, IFN- $\gamma$, IL- $1 \beta$, IL-6, IL-12, and IL-17) were expressed highly in the DSS group, but the antiinflammatory cytokine (IL-4) expression was low, and these changes were reversed by EFH treatment. Proinflammatory cytokines mediate cell infiltration [44], whereas antiinflammatory cytokines are involved in tissue growth, repair, and anti-inflammatory responses [45]. Therefore, EFH suppressed the NF- $\kappa \mathrm{B}$ pathway to upregulate the antiinflammatory cytokine but downregulate proinflammatory cytokines, which formed an anti-inflammatory environment to improve colon tissue repair.

Activated NF- $\kappa \mathrm{B}$ transcriptionally expresses many proteins involved in the initiation of signal transduction cascades, especially COX-2 and iNOS [46]. In fact, iNOS works synergistically with COX-2 to release cytokines and contribute to the development of inflammatory reactions [47]. As expected, high expressions of iNOS and COX-2 in the DSS group were detected, and these were reduced with EFH treatment. The anti-inflammatory effects of EFH might not only reduce the release of inflammatory factors but also downregulate iNOS and COX-2 mRNA levels.

Furthermore, two approved drugs (SASP and AZA) were used to more comprehensively evaluate the efficacy of EFH. As shown in the graphical abstract, the two drugs are effective treatments for UC as measured clinically and 
pharmacologically [48]. SASP can decompose to 5-ASA to inhibit the synthesis and release of PGE2 (related to COX2 ), and AZA can suppress the NF- $\kappa$ B pathway through immune suppression. The results of this study, comparing EFH effects with those of SASP and AZA, suggest that EFH may be a potential better therapeutic agent than SASP or AZA for the treatment of UC.

\section{Conclusions}

Our study shows novel insights into the anti-inflammatory effects of EFH in DSS-induced colitis in mice. The protective effects of EFH may be associated with the regulation of the gut microbiota, suppression of the NF- $\kappa \mathrm{B}$ pathway, and subsequent downregulation of inflammatory mediators. Our investigation provides experimental evidence for the pharmaceutical application of EFH in the treatment of UC and encourages further study of this fruit.

\section{Abbreviations}

$\begin{array}{ll}\text { AZA: } & \text { Azathioprine } \\ \text { COX-2: } & \text { Cyclooxygenase-2 } \\ \text { DSS: } & \text { Dextran sulfate sodium } \\ \text { EFH: } & \text { Extracts of Heritiera littoralis fruit } \\ \text { LC-ESI-MS: } & \text { Liquid chromatography-electrospray ioniza- } \\ & \text { tion mass spectrometry } \\ \text { iNOS: } & \text { Inducible nitric oxide synthase } \\ \text { IFN- } \gamma: & \text { Interferon- } \gamma \\ \text { IL-1 } \beta: & \text { Interleukin-1 } \beta \\ \text { IL-4: } & \text { Interleukin-4 } \\ \text { IL-12: } & \text { Interleukin-12 } \\ \text { IL-17: } & \text { Interleukin-17 } \\ \text { MPO: } & \text { Myeloperoxidase } \\ \text { NF- } \kappa \text { B: } & \text { Nuclear factor-kappa B } \\ \text { UC: } & \text { Ulcerative colitis } \\ \text { SASP: } & \text { Sulfasalazine } \\ \text { TNF- } \alpha: & \text { Tumor necrosis factor- } \alpha .\end{array}$

\section{Data Availability}

The data used to support the findings of this study are available from the corresponding author upon request.

\section{Conflicts of Interest}

The authors declare that there is no conflict of interests with respect to this article.

\section{Authors' Contributions}

G.L., N.X., J.L., and Y.W. performed the experiments. G.L. and M.L. analyzed the data. G.L., M.L., and X.W. wrote the paper. Q.Z., J.C., C.G., and Z.S. conceived and designed the experiments. Guosheng Lin and Minyao Li contributed equally to this work.

\section{Acknowledgments}

This work was supported by grants from the National Key R\&D Program of China (No. 2017YFC0506200), Guangdong Forestry Science and Technology Innovation Program (No. 2016KJCX026), Science and Technology Development Special Project of Guangdong Province (No. 2017A050506044), Guangdong Provincial Department of Education Feature Innovation Project (No. 2016KTSCX018), and Science and Technology Project of Guangzhou (No. 201704030028).

\section{References}

[1] D. Turner, A. Levine, J. C. Escher et al., "Management of pediatric ulcerative Colitis," Journal of Pediatric Gastroenterology, vol. 55, no. 3, pp. 340-361, 2012.

[2] S. Kaliyeva, N. Simohina, Y. Yukhnevich, Z. Myasnikova, and Y. Myasnikov, "Pharmacoeconomic assessment of biological therapy of ulcerative colitis," Value in Health, vol. 21, p. S84, 2018.

[3] M. Sałaga, H. Zatorski, M. Sobczak, C. Chen, and J. Fichna, "Chinese herbal medicines in the treatment of IBD and colorectal cancer: a review," Current Treatment Options in Oncology, vol. 15, no. 3, pp. 405-420, 2014.

[4] A. Himes-Cornell, L. Pendleton, and P. Atiyah, "Valuing ecosystem services from blue forests: a systematic review of the valuation of salt marshes, sea grass beds and mangrove forests," Ecosystem Services, vol. 30, pp. 36-48, 2018.

[5] H. Wangensteen, L. Klarpås, M. Alamgir, A. Samuelsen, and K. Malterud, "Can scientific evidence support using Bangladeshi traditional medicinal plants in the treatment of diarrhoea? A review on seven plants," Nutrients, vol. 5, no. 5, pp. 1757-1800, 2013.

[6] S. Tewtrakul, P. Tansakul, C. Daengrot, C. Ponglimanont, and C. Karalai, "Anti-inflammatory principles from Heritiera littoralis bark," Phytomedicine, vol. 17, no. 11, pp. 851-855, 2010.

[7] R. Christopher, S. S. Nyandoro, M. Chacha, and C. B. de Koning, "A new cinnamoylglycoflavonoid, antimycobacterial and antioxidant constituents from Heritiera littoralis leaf extracts," Natural Product Research, vol. 28, no. 6, pp. 351-358, 2014.

[8] L. Ge, Y. Li, K. Yang, and Z. Pan, "Chemical constituents of the leaves of Heritiera littoralis," Chemistry of Natural Compounds, vol. 52, no. 4, pp. 702-703, 2016.

[9] M.-Y. Li, Q. Xiao, J.-Y. Pan, and J. Wu, "Natural products from semi-mangrove flora: source, chemistry and bioactivities," Natural Product Reports, vol. 26, no. 2, pp. 281-298, 2009.

[10] Z.-H. Shen, C.-X. Zhu, Y.-S. Quan et al., "Relationship between intestinal microbiota and ulcerative colitis: mechanisms and clinical application of probiotics and fecal microbiota transplantation," World Journal of Gastroenterology, vol. 24, no. 1, pp. 5-14, 2018.

[11] K. M. Sakthivel and C. Guruvayoorappan, "Amentoflavone inhibits iNOS, COX-2 expression and modulates cytokine profile, NF- $\kappa \mathrm{B}$ signal transduction pathways in rats with ulcerative colitis," International Immunopharmacology, vol. 17, no. 3, pp. 907-916, 2013.

[12] M. A. Alghamdi, A. M. Hussein, L. N. AL-Eitan et al., "Possible mechanisms for the renoprotective effects of date palm fruits and seeds extracts against renal ischemia/reperfusion injury in rats," Biomedicine \& Pharmacotherapy, vol. 130, p. 110540, 2020. 
[13] A.-A. Neamtu, R. Szoke-Kovacs, E. Mihok et al., "Bilberry (Vaccinium myrtillus L.) extracts comparative analysis regarding their phytonutrient profiles, antioxidant capacity along with the in vivo rescue effects tested on a Drosophila melanogaster high-sugar diet model," Antioxidants, vol. 9, no. 11, p. 1067, 2020.

[14] S. Wirtz, C. Neufert, B. Weigmann, and M. F. Neurath, "Chemically induced mouse models of intestinal inflammation," Nature Protocols, vol. 2, pp. 541-546, 2007.

[15] Y.-F. Huang, J.-T. Zhou, C. Qu et al., "Anti-inflammatory effects of Brucea javanica oil emulsion by suppressing NF- $\kappa \mathrm{B}$ activation on dextran sulfate sodium-induced ulcerative colitis in mice," Journal of Ethnopharmacology, vol. 198, pp. 389-398, 2017.

[16] M. Murano, K. Maemura, I. Hirata et al., "Therapeutic effect of intracolonically administered nuclear factor kappa B (p65) antisense oligonucleotide on mouse dextran sulphate sodium (DSS)-induced colitis," Clinical Experimental Immunology, vol. 120, no. 1, pp. 51-58, 2000

[17] J. Li, W. Zhong, W. Wang et al., "Ginsenoside metabolite compound $\mathrm{K}$ promotes recovery of dextran sulfate sodiuminduced colitis and inhibits inflammatory responses by suppressing NF- $\kappa \mathrm{B}$ activation," PLoS One, vol. 9, no. 2, article e87810, 2014.

[18] X. Wang, G. Dong, X. Liu et al., "Poly- $\gamma$-glutamic acidproducing bacteria reduced $\mathrm{Cd}$ uptake and effected the rhizosphere microbial communities of lettuce," Journal of Hazardous Materials, vol. 398, article 123146, 2020.

[19] E. Mazzon, P. Guigues, and J.-P. Habas, "Biobased structural epoxy foams derived from plant-oil: formulation, manufacturing and characterization," Industrial Crops and Products, vol. 144, article 111994, 2020.

[20] D. M. Jia, Y. P. Li, Y. J. Li, Y. G. Li, and C. H. Li, "Fixed bed adsorption of 2-naphthalenesulfonic acid from aqueous solution by composite resin," Water Environment Research, vol. 86, no. 2, pp. 99-103, 2014.

[21] N. Shen, Z. Liang, Q. Liu et al., “Antifungal secondary metabolites isolated from mangrove rhizosphere soil-derived penicillium fungi," Journal of Ocean University of China, vol. 19, no. 3, pp. 717-721, 2020.

[22] H. Zeitoun, Z. Khan, K. Banerjee, D. Salameh, and R. Lteif, "antityrosinase activity of Combretum micranthum, Euphorbia hirta and Anacardium occidentale plants: ultrasound assisted extraction optimization and profiling of associated predominant metabolites," Molecules, vol. 25, no. 11, p. 2684, 2020.

[23] M. Yin, J. Wang, H. Huang, Q. Huang, Z. Fu, and Y. Lu, “Analysis of flavonoid compounds by terahertz spectroscopy combined with chemometrics," ACS Omega, vol. 5, no. 29, pp. 18134-18141, 2020.

[24] X. Cao, R. Bi, J. Hao et al., "A study on the protective effects of taxifolin on human umbilical vein endothelial cells and THP-1 cells damaged by hexavalent chromium: a probable mechanism for preventing cardiovascular disease induced by heavy metals," Food \& Function, vol. 11, no. 5, pp. 3851-3859, 2020.

[25] S. Safe, "Carbidopa: a selective Ah receptor modulator (SAhRM)," Biochemical Journal, vol. 474, no. 22, pp. 37633765, 2017.

[26] S. Uruma, Y. Shibata, D. Takemoto, and K. Kawakita, "N,Ndimethylsphingosine, an inhibitor of sphingosine kinase, induces phytoalexin production and hypersensitive cell death of Solanaceae plants without generation of reactive oxygen species," Journal of General Plant Pathology, vol. 75, no. 4, pp. 257-266, 2009.

[27] C. Sarikurkcu, "Anthemis chia: biological capacity and phytochemistry," Industrial Crops and Products, vol. 153, p. 112578, 2020.

[28] J. Teng, C. Yan, W. Zeng, Y. Zhang, Z. Zeng, and Y. Huang, "Purification and characterization of theobromine synthase in a theobromine-enriched wild tea plant (Camellia gymnogyna Chang) from Dayao Mountain, China," Food Chemistry, vol. 311, p. 125875, 2020.

[29] F. Han, H. Zhang, X. Xia et al., "Porcine $\beta$-defensin 2 attenuates inflammation and mucosal lesions in dextran sodium sulfate-induced colitis," Journal of Immunology, vol. 194, no. 4, pp. 1882-1893, 2015.

[30] H. Herfarth, K. Brand, H. C. Rath, G. Rogler, J. Schölmerich, and W. Falk, "Nuclear factor-kappa B activity and intestinal inflammation in dextran sulphate sodium (DSS)-induced colitis in mice is suppressed by gliotoxin," Clinical Experimental Immunology, vol. 120, pp. 59-65, 2000.

[31] A. Leonard, M. W. Millar, S. A. Slavin et al., "Critical role of autophagy regulator Beclin1 in endothelial cell inflammation and barrier disruption," Cellular Signalling, vol. 61, pp. 120129, 2019.

[32] Å. Håkansson, N. Tormo-Badia, A. Baridi et al., "Immunological alteration and changes of gut microbiota after dextran sulfate sodium (DSS) administration in mice," Clinical Experimental Medicine, vol. 15, no. 1, pp. 107-120, 2015.

[33] X.-J. Zhang, Z.-W. Yuan, C. Qu et al., "Palmatine ameliorated murine colitis by suppressing tryptophan metabolism and regulating gut microbiota," Pharmacological Research, vol. 137, pp. 34-46, 2018.

[34] Y.-h. Li, H.-t. Xiao, D.-d. Hu et al., "Berberine ameliorates chronic relapsing dextran sulfate sodium-induced colitis in C57BL/6 mice by suppressing Th17 responses," Pharmacological Research, vol. 110, pp. 227-239, 2016.

[35] W. Chen, W. Deng, and S. Chen, "Inactivation of Nf-kappa b pathway by taxifolin attenuates sepsis-induced acute lung injury," Current Topics in Nutraceutical Research, vol. 18, pp. 176-182, 2020.

[36] L. Wang, Y. Wu, L. Zhuang et al., "Puerarin prevents high-fat diet-induced obesity by enriching Akkermansia muciniphila in the gut microbiota of mice," PLoS One, vol. 14, no. 6, p. e0218490, 2019.

[37] I. Okayasu, S. Hatakeyama, M. Yamada, T. Ohkusa, Y. Inagaki, and R. Nakaya, "A novel method in the induction of reliable experimental acute and chronic ulcerative colitis in mice," Gastroenterology, vol. 98, no. 3, pp. 694-702, 1990.

[38] C. T. Peterson, V. Sharma, L. Elmén, and S. N. Peterson, "Immune homeostasis, dysbiosis and therapeutic modulation of the gut microbiota," Clinical Experimental Immunology, vol. 179, no. 3, pp. 363-377, 2015.

[39] C. L. Hager and M. A. Ghannoum, "The mycobiome: role in health and disease, and as a potential probiotic target in gastrointestinal disease," Digestive and Liver Disease, vol. 49, no. 11, pp. 1171-1176, 2017.

[40] T. Rokkas, J. P. Gisbert, Y. Niv, and C. O’Morain, “The association between Helicobacter pylori infection and inflammatory bowel disease based on meta-analysis," United European Gastroenterology Journal, vol. 3, no. 6, pp. 539-550, 2015.

[41] T. Horiuchi, H. Mitoma, S.-I. Harashima, H. Tsukamoto, and T. Shimoda, “Transmembrane TNF-alpha: structure, function 
and interaction with anti-TNF agents," Rheumatology, vol. 49, no. 7, pp. 1215-1228, 2010.

[42] D. N. Jackson and A. L. Theiss, "Gut bacteria signaling to mitochondria in intestinal inflammation and cancer," Gut Microbes, vol. 11, no. 3, pp. 285-304, 2020.

[43] P. Ó. Cuív, T. de Wouters, R. Giri et al., "The gut bacterium and pathobiont Bacteroides vulgatus activates NF- $\kappa \mathrm{B}$ in a human gut epithelial cell line in a strain and growth phase dependent manner," Anaerobe, vol. 47, pp. 209-217, 2017.

[44] W. Liu, W. Guo, L. Guo et al., "Andrographolide sulfonate ameliorates experimental colitis in mice by inhibiting Th1/Th17 response," International Immunopharmacology, vol. 20, no. 2, pp. 337-345, 2014.

[45] I. G. Luzina, A. D. Keegan, N. M. Heller, G. A. W. Rook, T. Shea-Donohue, and S. P. Atamas, "Regulation of inflammation by interleukin-4: a review of "alternatives"," Journal of Leukocyte Biology, vol. 92, no. 4, pp. 753-764, 2012.

[46] L. Chen, H. Teng, T. Fang, and J. Xiao, “Agrimonolide from Agrimonia pilosa suppresses inflammatory responses through down-regulation of COX-2/iNOS and inactivation of NF- $\kappa \mathrm{B}$ in lipopolysaccharide-stimulated macrophages," Phytomedicine, vol. 23, no. 8, pp. 846-855, 2016.

[47] S. H. Itzkowitz, "Molecular biology of dysplasia and cancer in inflammatory bowel disease," Gastroenterology Clinics of North America, vol. 35, no. 3, pp. 553-571, 2006.

[48] A. O. M. C. Damião, M. F. C. de Azevedo, A. de Sousa Carlos, M. Y. Wada, T. V. M. Silva, and F. de Castro Feitosa, "Conventional therapy for moderate to severe inflammatory bowel disease: a systematic literature review," World Journal of Gastroenterology, vol. 25, no. 9, pp. 1142-1157, 2019. 